\title{
Hydrodynamic analysis of marine multibody systems by a nonlinear coupled model
}

\author{
X. Feng ${ }^{\mathrm{a}}$, W. Bai ${ }^{\mathrm{b}, *}$ \\ ${ }^{a}$ Department of Civil and Environmental Engineering, National University of Singapore, Kent Ridge, Singapore 117576, \\ Singapore \\ ${ }^{b}$ School of Computing, Mathematics and Digital Technology, Manchester Metropolitan University, Chester Street, \\ Manchester M1 5GD, UK
}

\begin{abstract}
This study investigates the hydrodynamic performance of two freely floating or interconnected barges. A nonlinear decomposition model in the framework of potential flow is employed to simulate the wave-body interactions in the time domain. This piece of work focuses on the accurate calculation of hydrodynamic forces and motions of a multibody floating system. The hydrodynamic forces are indirectly calculated through an auxiliary function approach, which is extended to application of two floating bodies in the present study. The resultant coupled motion equations for two bodies clearly demonstrate the influence of one body on the other. To incorporate an interconnection between two floating bodies, a constraint matrix method is developed where the connection constraints are mathematically represented by a constraint matrix. The constraint forces in the connection are solved in the modified motion equations. The newly formulated coupled auxiliary function and constraint matrix approaches for two bodies with and without interconnections are validated by comparisons of first-order response against linear frequency-domain models. Two types of interconnections, namely, the rigid connection and the middle-hinge connection, are examined in both beam sea and head sea to demonstrate the effectiveness of the proposed method. Coupling effects of different connection scenarios on body motions as well as the influence on constraint forces are discussed. Furthermore, simulations using relatively steeper waves are performed and compared with mild wave cases. The nonlinear effect is highlighted by decomposing the higher harmonic components of nonlinear responses. Keywords: Multibody, Auxiliary function, Coupling, Side-by-side, Constraint matrix, nonlinearity
\end{abstract}

\section{Introduction}

The multibody hydrodynamics has long been studied in the discipline of ocean engineering. The complexity of multibody hydrodynamics lies in not only the structure-induced severe wave field but also the coupled multibody dynamics if the bodies are interconnected. To date, we have seen increasingly more floating multibody systems across the global waters, and they are becoming dramatically crucial in terms of economy and human life. For instance, permanent structures include floating LNG terminal clusters,

\footnotetext{
${ }^{*}$ Corresponding author

Email address: w.bai@hotmail.com (W. Bai)
} 
multiple large floating blocks for the purpose of land claim; and some temporary offshore multibody systems include loading/offloading operations between LNG carrier and FPSO, ship-to-ship cargo transfer, and marine lifting/installation. More reliable tools need to be developed to accurately predict the hydrodynamic behaviors of such complicated coupled systems, and to produce knowledge on multibody hydrodynamics.

Linear frequency-domain potential flow models for dealing with wave-body interactions were profoundly developed in various research groups a few decades ago and remain dominantly utilized in the industry. Based on the linear frequency-domain models, some works have been done concerning the multibody hydrodynamics. Earlier, Ohkushu (1974), Van Oortmerssen (1979), Loken (1981), Kodan (1984), and Fang and Kim (1986) investigated two adjacent bodies in waves without connection in-between, which highlighted the influence of multibody hydrodynamic interactions. More recently, Kashiwagi et al. (2005) studied two side-by-side ships, with a focus on the wave drift forces and moments. Fairly good agreements were obtained between calculations and measurements for both first order forces and second order drift forces. Nevertheless, these early works did not consider any interconnection between the floating bodies, and the motion equation of each body is individually solved in the frequency domain.

In the cases where the adjacent bodies are interconnected, possibly by hinges or moorings, a key concern is the dynamic behavior of the interconnected bodies and more importantly the motion coupling effect due to the interconnection. Newman (1994) introduced a mode expansion method to compute the motions of simply connected (rigid or hinged) floating bodies as well as the motions of the connection, within the frame work of linear frequency-domain model. In this method, additional motion modes due to the deformation of the simple-link multibody system are solved together with the motion modes of the bodies. It is generally effective in terms of considering the dynamic coupling effect between the bodies, as adopted in Lee and Newman (2000) and Taghipour and Moan (2008). A similar concept of modelling connection is utilized in the analysis of hydroelastic response of flexible floating interconnected structures. For instance, Fu et al. (2007) presented a hydroelastic model by taking into account rigid/hinge modes and studied two flexible interconnected floating plates. Good agreements were found between their numerical results and experiments for a special case with high stiffness connection (equivalent to rigid). Wang et al. (2010) employed the mode expansion method presented by Newman (1994) to model the structure deflection and investigated the hydroelastic response of very large floating structure with a hinge or semi-rigid line connection. Nevertheless, the mode expansion method is mainly suitable to very simply linked bodies where the system can be regarded as a single deformable body. For systems with complex constraints, the connection motions may not be modelled as expanded modes. In addition, frequency-domain calculations in any case present limited information for the hydrodynamic process of wave interaction with an interconnected floating system.

In modelling the dynamic response, a prevailing strategy is to firstly calculate wave forces and hydrodynamic coefficients (added mass and radiation damping coefficient) from a linear frequency-domain diffraction-radiation model, then the motion equations of multibody systems are solved in the time domain. This two-step strategy is favored nowadays in the industry, because it is quite convenient to add whatever 
extra external loading (mooring, fender, hawser and riser) into the motion equations to perform dynamic analysis. Applying this strategy, Koo and Kim (2005) simulated two moored vessels in side-by-side offloading operation in irregular waves by using a combined matrix method. They considered both vessel and mooring dynamics, as well as the coupled hydrodynamic coefficients. The sway and roll motions were found significantly influenced by the mechanical coupling effects of the two vessels. Hong et al. (2005) numerically and experimentally investigated three side-by-side moored vessels. They adopted a generalized mode concept to obtain their time-domain motion equations by expanding the transient motion equation of a single body which was described in Cummins (1962). This generalized mode approach was previously presented in Choi and Hong (2002). O'Cathain et al. (2008) modeled and analyzed a hinged-barge wave-energy system in the time domain by solving the Newton-Euler equations of motion and eliminating degrees of freedom associated with the hinge constraints. Again, by employing the hydrodynamic coefficients computed from a linear frequency-domain model DIFFRACT, Sun et al. (2011) implemented the motion equations with constraints by using Lagrange multipliers and studied a tank connected with an FLNG barge by rigid, hinge and spring-type connections. It was shown that the free surface elevation in the gap between the two vessels is obviously influenced by the behavior of the floating vessels and the constraint forces in the connections. A similar work concerning a multibody pier was also presented by Tajali and Shafieefar (2011). Nevertheless, at any rate simulations via the two-step approach do not reproduce the real hydrodynamic process of wave interaction with a multibody floating system. The reason is that the two-step approach requires the linear frequency-domain results which are essentially obtained at the equilibrium positions of the system.

The aim of this study is to develop an effective dynamic model capable of calculating responses of a multibody system with or without interconnections in the framework of three-dimensional fully nonlinear potential flow theory. Unlike the two-stage approach, we solve both the wave field and the body motions simultaneously in the time domain, which reproduces the real dynamic responses of the multibody floating system. Two main contributions of the newly developed model are: (a) extension of the formulation of the auxiliary function approach, which is used for indirect calculation of wave forces, in application of multiple floating bodies; and (b) development of a constraint matrix method for modeling interconnections between bodies. The newly derived motion equation system clearly demonstrates the hydrodynamic coupling effect of two floating bodies and the constraint effect on the floating system due to the interconnection. Furthermore, through simulations by employing this model, a better understanding of coupled hydrodynamic behavior of marine multibody systems with/without interconnections is created. The nonlinearity associated with free surface conditions is also identified by comparisons between mild and relatively steep wave conditions.

The organization of this paper is as follows. Section 2 briefly presents the governing equations of the fully nonlinear potential flow model as well as a wave decomposition strategy. Based on this model, we, for the first time, derive the formulation of the coupled auxiliary functions approach to indirectly calculate wave forces for the case of two floating bodies. In cases where the bodies are interconnected, we present a constraint matrix method for modelling the connections of various types, and the constraint forces in the 
connection are solved simultaneously with the coupled body motions. Section 5 simulates a case of two freely floating barges. The first order results, extracted from the present fully nonlinear time-domain simulations, are compared with the linear calculations, where satisfactory agreements are achieved. Section 6 simulates two side-by-side interconnected barges of a simple configuration. Validation is also performed by comparisons against a linear model in Newman (1994). Two types of connections are then modelled. The effect of each type of connection is discussed, where their advantages and disadvantages are compared. A great concern is the constraint forces in different types of connections which may determine the practical design. The nonlinear effect is finally investigated by comparisons of simulation results with different wave steepnesses. Concluding remarks are drawn in Section 7 .

\section{Mathematical formulation}

\subsection{Nonlinear decomposition model}

To simulate the problem of wave-body interactions, a numerical wave tank (NWT) is defined. The schematic diagram in Fig. 1 shows a circular wave tank, with two bodies floating in the tank. Two coordinate systems are defined in the model: a space-fixed global coordinate system Oxyz and a body-fixed local coordinate system $O^{\prime} x^{\prime} y^{\prime} z^{\prime}$. In the global coordinate system, the origin $O$ is placed on the undisturbed water surface at the center of the circular tank with its $z$-axis pointing upward; in the local coordinate system, the origin $O^{\prime}$ is fixed at the center of gravity $\mathbf{X}_{g}=\left(x_{g}, y_{g}, z_{g}\right)$ of the corresponding body. The local coordinate system is parallel to the global coordinate system when the floating bodies are at their equilibrium positions.

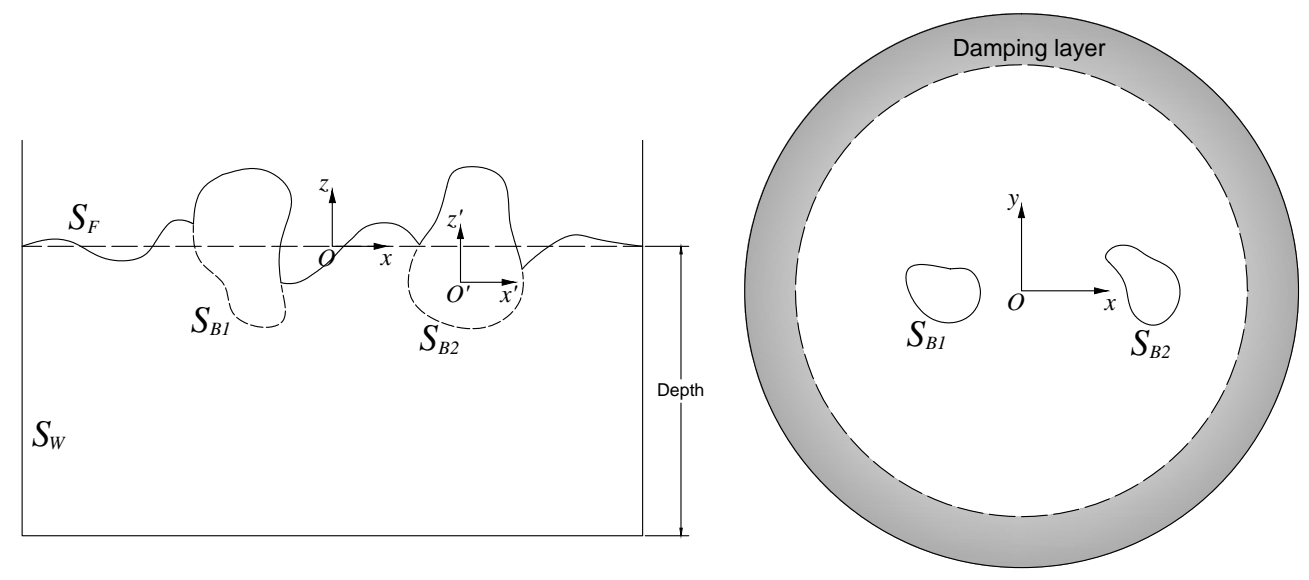

Figure 1: Definition of numerical tank coordinate system

Following the assumption that the fluid is incompressible and inviscid, and the flow irrotational, the water wave problem can be formulated in the framework of potential flow theory. A velocity potential 
$\phi(x, y, z, t)$ defined in the global coordinate system $O x y z$ satisfies the Laplace equation in the fluid domain $\Omega$, i.e. $\nabla^{2} \phi=0$. Instead of solving the potential $\phi$ directly, we adopt a wave decomposition strategy which separates the total wave into an incident and a scattered wave. Mathematically, the total velocity potential is rewritten as $\phi=\phi_{I}+\phi_{S}$ and the position of water particles on the free water surface is represented as $\mathbf{X}=\mathbf{X}_{I}+\mathbf{X}_{S}$, where the subscripts ' $I$ ' and ' $S$ ' indicate the quantities for incident and scattered waves respectively. A complete description of the decomposition model can be found in Feng and Bai (2015). Essentially, the incident wave field is prescribed and only the scattered wave component is solved. The velocity potential for the scattered wave satisfies the Laplace equation in the fluid domain $\boldsymbol{\Omega}$,

$$
\nabla^{2} \phi_{S}=0 \quad \text { in } \Omega
$$

On the free water surface $S_{F}$, the kinematic and dynamic boundary conditions for the scattered wave in the Lagrangian description are

$$
\begin{gathered}
\frac{D \mathbf{X}_{S}}{D t}=\nabla \phi-\nabla \phi_{I} \quad \text { on } \quad S_{F} \\
\frac{D \phi_{S}}{D t}=-g z_{S}+\frac{1}{2} \nabla \phi \cdot \nabla \phi-\frac{1}{2} \nabla \phi_{I} \cdot \nabla \phi_{I} \quad \text { on } \quad S_{F}
\end{gathered}
$$

where $\frac{D}{D t}=\frac{\partial}{\partial t}+v \cdot \nabla$ is the material derivative at each fluid particle, $z_{S}$ the scattered wave elevation, $t$ the time and $g$ the gravitational acceleration. Note that both the total flow velocity $\nabla \phi$ and the incident velocity component $\nabla \phi_{I}$ are calculated on the real time free water surface $S_{F}$. On the wetted body surface $S_{B}$, the boundary condition is specified as

$$
\frac{\partial \phi_{S}}{\partial \mathbf{n}}=\mathbf{V}_{\mathbf{n}}-\frac{\partial \phi_{I}}{\partial \mathbf{n}} \quad \text { on } \quad S_{B}
$$

where $\mathbf{n}$ is the unit normal vector pointing out of the fluid domain in the global coordinate system, and $\mathbf{V}_{\mathbf{n}}$ the normal velocity of the body surface. Based on the small angular motion assumption, the normal velocity of a three-dimensional body about its center of gravity $\mathbf{X}_{g}$ can be expressed as

$$
\mathbf{V}_{\mathbf{n}}=\left[\dot{\boldsymbol{\xi}}-\dot{\boldsymbol{\alpha}} \times\left(\mathbf{X}-\mathbf{X}_{g}\right)\right] \cdot \mathbf{n}
$$

where $\boldsymbol{\xi}=\left(\xi_{x}, \xi_{y}, \xi_{z}\right)$ is a translational vector denoting the displacements of surge, sway, and heave, while $\boldsymbol{\alpha}=\left(\alpha_{x}, \alpha_{y}, \alpha_{z}\right)$ is a rotational vector denoting the motions of roll, pitch and yaw. On the tank walls $S_{W}$, the impermeable condition shall be imposed

$$
\frac{\partial \phi_{S}}{\partial \mathbf{n}}=-\frac{\partial \phi_{I}}{\partial \mathbf{n}}
$$

Quantities associated with the prescribed incident wave are explicitly evaluated. In this study we adopt a fully nonlinear stream function model to describe the incident waves. Formulation and implementation of the stream function model can be found in Fenton (1988).

At the beginning of simulations, a ramp function is applied on the prescribed incident wave to ensure that the incident flow develops smoothly from the still water surface to a fully periodic wave. We adopt the same 
cosine ramp function as in Bai and Eatock Taylor (2006) and choose a ramping time of two incident wave periods, which has been well tested in Feng and Bai (2015).In Fig. 1 a damping zone is placed near the tank wall to absorb the far-field wave energy, so that any reflection from the tank wall can be prevented. In this regard the free surface boundary conditions Eqs. (2) and (3) need to be modified accordingly to include the damping layer, see Ferrant (1993) for more details. In the present decomposition model, only the scattered wave field near the tank wall is to be damped out.

A higher-order boundary element method (HOBEM) is employed to solve the reformulated boundary value problem for the scattered wave. Quadratic isoparametric elements are utilized to discrete the fluid domain boundaries. In particular, structured 8-node quadrilateral elements are distributed on the vertical patches including the tank wall and body vertical surfaces. On the free surface and body bottom surfaces, unstructured 6-node triangular elements are generated by a Delaunay triangulation method. The boundary integral equation in the HOBEM is formulated by the Green's second identity, which is to evaluate integration over the computational surface. By discretization through a shape function, a set of algebraic linear equations can be obtained, which are solved by an efficient open source LAPACK solver. A thorough introduction on the numerical implementation of the HOBEM, including mesh generation, time marching, numerical beach and interpolation, was presented in Bai and Eatock Taylor (2006).

\subsection{Calculation of hydrodynamic forces}

Hydrodynamic forces on the bodies can be calculated by integrating Bernoulli's pressure over the instantaneous wetted surface. Bernoulli's pressure reads

$$
p=-\rho\left(\phi_{t}+\frac{1}{2} \nabla \phi \cdot \nabla \phi+g z\right)
$$

where $\rho$ is the density of fluid, and $\phi_{t}=\frac{\partial \phi}{\partial t}$. The hydrodynamic forces $\mathbf{F}=\left(F_{1}, F_{2}, F_{3}\right)$ and the corresponding moments $\mathbf{M}=\left(F_{4}, F_{5}, F_{6}\right)$ are the integrations of pressure over the wetted body surface, which read

$$
F_{i}=\int_{S_{B}} p n_{i} \mathrm{~d} S \quad(i=1,2, \cdots, 6)
$$

or

$$
F_{i}=-\rho \int_{S_{B}} \phi_{t} n_{i} \mathrm{~d} S-\rho \int_{S_{B}}\left(\frac{1}{2} \nabla \phi \cdot \nabla \phi+g z\right) n_{i} \mathrm{~d} S \quad(i=1,2, \cdots, 6)
$$

where $n_{i}$ are the six components of the normal unit vector $\mathbf{n}$. At each time step, after the velocity potential is successfully solved through the HOBEM, it is straightforward to calculate the velocity components $\nabla \phi$ on the body surface. However, the calculation of the time derivative of velocity potential, $\phi_{t}$, is crucial to obtain the accurate forces at each time step. A simple backward difference scheme is inaccurate and prone to instabilities especially in the case of freely moving bodies (Tanizawa, 1995).

In the literature, several methods have been developed to tackle the problem. An acceleration potential method was presented by Tanizawa (1995), who defined an acceleration potential $\Phi$ in the fluid domain as $\Phi=\phi_{t}+\frac{1}{2} \nabla \phi \cdot \nabla \phi$. This method involves the evaluation of the surface local curvature, which makes it 
extremely difficult in 3D cases where the body geometry is rather complicated (though this may not be a problem in 2D simulations). Vinje and Brevig (1981) introduced a mode decomposition method which decomposed the time derivative $\phi_{t}$ into modes corresponding to each degree of freedom of body motions, and a mode of acceleration due to velocity field. Each mode can be obtained by calculating its respective boundary integral equation. The mode decomposition method was adopted by Cointe et al. (1990) and Koo and Kim (2004) in their 2D numerical wave tanks. An auxiliary function approach for indirect calculation of wave force was proposed by $\mathrm{Wu}$ and Eatock Taylor (2003). In this approach, they defined a set of auxiliary functions $\psi_{i}$ which correspond to each degree of freedom of the body motions. Instead of calculating the time derivative $\phi_{t}$ itself, they evaluated its integral over the wetted body surface as a whole term, $\int_{S_{B}} \phi_{t} n_{i} \mathrm{~d} S$, which is exactly required in calculating the forces in Eq. (9). To compute the auxiliary functions, extra boundary value problems (BVPs) have to be solved. As the auxiliary functions share the same coefficient matrix with the velocity potential when proper boundary conditions are imposed, they are solved simultaneously with the potential, and not much additional computational effort is needed for solving these extra BVPs for the auxiliary functions.

In this study, we employ the auxiliary function approach for calculating the wave forces. However, the formula in Wu and Eatock Taylor (2003) are only applicable for a single body case. In the cases where multiple bodies are considered, one cannot simply use the same auxiliary functions for each body, as this may omit the coupled interactions between multiple bodies. We extend the application of the auxiliary function approach to multibody cases. The following section derives in detail the formulation of coupled auxiliary functions, which is presented for the first time in the literature.

\section{Coupled auxiliary functions approach}

In this work, we take the case of two bodies as an example in application of the auxiliary function approach, whereas similar formulas can be obtained for more bodies. We define 12 auxiliary functions $\psi_{i}(i=1,2, \cdots, 12)$, where $\psi_{i}(i=1,2, \cdots, 6)$ correspond to motions of Body 1 and $\psi_{i}(i=7,8, \cdots, 12)$ to motions of Body 2. Additional boundary value problems for the 12 auxiliary functions have to be formulated in order to solve these $\psi_{i}$. Naturally we let the auxiliary functions satisfy the Laplace equation in the fluid domain

$$
\nabla^{2} \psi_{i}=0
$$

The boundary conditions for the auxiliary functions must be adequately imposed, which determines the complexity and resolvability of the boundary value problems. The entire computational boundary includes $S=S_{W}+S_{B 1}+S_{B 2}+S_{F}$. The following boundary conditions are imposed.

On the tank side wall $S_{W}$ :

$$
\frac{\partial \psi_{i}}{\partial n}=0 \quad(i=1,2, \cdots, 12)
$$


On the surface of Body $1 S_{B 1}$ :

$$
\begin{aligned}
& \frac{\partial \psi_{i}}{\partial n}=n_{1, i} \quad(i=1,2, \cdots, 6) \\
& \frac{\partial \psi_{i}}{\partial n}=0 \quad(i=7,8, \cdots, 12)
\end{aligned}
$$

where $n_{1, i}(i=1,2, \cdots, 6)$ are the six components of the normal unit vector $\mathbf{n}_{\mathbf{1}}$ on Body 1 .

On the surface of Body $2 S_{B 2}$ :

$$
\begin{gathered}
\frac{\partial \psi_{i}}{\partial n}=0 \quad(i=1,2, \cdots, 6) \\
\frac{\partial \psi_{i}}{\partial n}=n_{2, i-6} \quad(i=7,8, \cdots, 12)
\end{gathered}
$$

where $n_{2, i}(i=1,2, \cdots, 6)$ are the six components of the normal unit vector $\mathbf{n}_{2}$ on Body 2 .

On the free surface $S_{F}$ :

$$
\psi_{i}=0 \quad(i=1,2, \cdots, 12)
$$

The mixed boundary value problems for each of the 12 auxiliary functions are essentially similar to that for the velocity potential hence solved simultaneously with the velocity potential. Once these boundary value problems are solved, we have obtained $\psi_{i}$ and $\frac{\partial \psi_{i}}{\partial n}$ on all the boundaries. A relationship between $\psi_{i}$ and $\phi_{t}$ can be constructed via Green's second identity as

$$
\iint_{S}\left(\phi_{t} \frac{\partial \psi_{i}}{\partial n}-\psi_{i} \frac{\partial \phi_{t}}{\partial n}\right) \mathrm{d} S=0
$$

On the free surface $S_{F}$, the dynamic boundary condition gives

$$
\phi_{t}=-\left(g z+\frac{1}{2} \nabla \phi \cdot \nabla \phi\right)
$$

On each body surface, Wu and Eatock Taylor (2003) have shown that

$$
\frac{\partial \phi_{t}}{\partial n}=\left[\ddot{\boldsymbol{\xi}}+\ddot{\boldsymbol{\alpha}} \times\left(\mathbf{X}-\mathbf{X}_{g}\right)\right] \cdot \mathbf{n}-\dot{\boldsymbol{\xi}} \cdot \frac{\partial \nabla \phi}{\partial n}+\dot{\boldsymbol{\alpha}} \cdot \frac{\partial}{\partial n}\left[\left(\mathbf{X}-\mathbf{X}_{g}\right) \times(\dot{\boldsymbol{\xi}}-\nabla \phi)\right]
$$

where $\mathbf{A}=[\ddot{\boldsymbol{\xi}}, \ddot{\boldsymbol{\alpha}}]^{T}$ is the acceleration of the corresponding body, which is unknown before the wave forces and body motions are solved. On the fixed tank wall $S_{W}$, the condition becomes

$$
\frac{\partial \phi_{t}}{\partial n}=0
$$

We rewrite Green's second identity Eq. (17) in a separated form as

$$
\begin{gathered}
\int_{S_{W}} \phi_{t} \frac{\partial \psi_{i}}{\partial n} \mathrm{~d} S+\int_{S_{B 1}} \phi_{t} \frac{\partial \psi_{i}}{\partial n} \mathrm{~d} S+\int_{S_{B 2}} \phi_{t} \frac{\partial \psi_{i}}{\partial n} \mathrm{~d} S+\int_{S_{F}} \phi_{t} \frac{\partial \psi_{i}}{\partial n} \mathrm{~d} S= \\
\int_{S_{W}} \psi_{i} \frac{\partial \phi_{t}}{\partial n} \mathrm{~d} S+\int_{S_{B 1}} \psi_{i} \frac{\partial \phi_{t}}{\partial n} \mathrm{~d} S+\int_{S_{B 2}} \psi_{i} \frac{\partial \phi_{t}}{\partial n} \mathrm{~d} S+\int_{S_{F}} \psi_{i} \frac{\partial \phi_{t}}{\partial n} \mathrm{~d} S
\end{gathered}
$$


We now substitute the boundary conditions for $\psi_{i}$ and $\phi_{t}$ into the above equation. As the boundary conditions on Body 1 and Body 2 surfaces for $\psi_{i}(i=1, \ldots, 6)$ and $\psi_{i}(i=7, \ldots, 12)$ are different, two sets of equations are obtained

$$
\begin{array}{r}
\int_{S_{B 1}} \phi_{t} n_{1, i} \mathrm{~d} S=\int_{S_{F}}\left(g z+\frac{1}{2} \nabla \phi \cdot \nabla \phi\right) \cdot \frac{\partial \psi_{i}}{\partial n} \mathrm{~d} S+\int_{S_{B 1}} \psi_{i} \frac{\partial \phi_{t}}{\partial n} \mathrm{~d} S+\int_{S_{B 2}} \psi_{i} \frac{\partial \phi_{t}}{\partial n} \mathrm{~d} S \\
(i=1,2, \cdots, 6) \\
\int_{S_{B 2}} \phi_{t} n_{2, i-6} \mathrm{~d} S=\int_{S_{F}}\left(g z+\frac{1}{2} \nabla \phi \cdot \nabla \phi\right) \cdot \frac{\partial \psi_{i}}{\partial n} \mathrm{~d} S+\int_{S_{B 1}} \psi_{i} \frac{\partial \phi_{t}}{\partial n} \mathrm{~d} S+\int_{S_{B 2}} \psi_{i} \frac{\partial \phi_{t}}{\partial n} \mathrm{~d} S \\
(i=7,8, \cdots, 12)
\end{array}
$$

where $\frac{\partial \phi_{t}}{\partial n}$ on the body surface is calculated by Eq. 19 which includes the respective body acceleration $A_{i}^{(1)}$ or $A_{i}^{(2)}$. After substituting $\frac{\partial \phi_{t}}{\partial n}$, for simplification we rewrite Eqs. 22 and 23 as

$$
\begin{gathered}
\int_{S_{B 1}} \phi_{t} n_{1, i} \mathrm{~d} S=\Gamma_{i}^{F}+C_{i j}^{(1)} \cdot A_{i}^{(1)}+E_{i}^{(1)}+D_{i j}^{(1)} \cdot A_{i}^{(2)}+E_{i}^{(2)} \quad(i=1,2, \cdots, 6) \\
\int_{S_{B 2}} \phi_{t} n_{2, i-6} \mathrm{~d} S=\Gamma_{i}^{F}+C_{i j}^{(2)} \cdot A_{i-6}^{(2)}+E_{i}^{(1)}+D_{i j}^{(2)} \cdot A_{i-6}^{(1)}+E_{i}^{(2)} \quad(i=7,8, \cdots, 12)
\end{gathered}
$$

where

$$
\begin{aligned}
\Gamma_{i}^{F} & =\int_{S_{F}}\left(g z+\frac{1}{2} \nabla \phi \cdot \nabla \phi\right) \cdot \frac{\partial \psi_{i}}{\partial n} \mathrm{~d} S \quad(i=1,2, \cdots, 12) \\
C_{i j}^{(1)} & =\int_{S_{B 1}} \psi_{i} n_{j} \mathrm{~d} S \quad(i, j=1,2, \cdots, 6) \\
D_{i j}^{(1)} & =\int_{S_{B 2}} \psi_{i} n_{j} \mathrm{~d} S \quad(i, j=1,2, \cdots, 6) \\
C_{i j}^{(2)} & =\int_{S_{B 2}} \psi_{i} n_{j} \mathrm{~d} S \quad(i=7,8, \cdots, 12 ; j=1,2, \cdots, 6) \\
D_{i j}^{(2)} & =\int_{S_{B 1}} \psi_{i} n_{j} \mathrm{~d} S \quad(i=7,8, \cdots, 12 ; j=1,2, \cdots, 6) \\
E_{i}^{(1)} & =\int_{S_{B 1}} \psi_{i} \Theta^{(1)} \mathrm{d} S \quad(i=1,2, \cdots, 12) \\
E_{i}^{(2)} & =\int_{S_{B 2}} \psi_{i} \Theta^{(2)} \mathrm{d} S \quad(i=1,2, \cdots, 12) \\
\boldsymbol{\Theta}^{(k)} & =-\dot{\boldsymbol{\xi}}^{(k)} \cdot \frac{\partial \nabla \phi}{\partial n}+\dot{\boldsymbol{\alpha}}^{(k)} \cdot \frac{\partial}{\partial n}\left[\left(\mathbf{X}-\mathbf{X}_{g}^{(k)}\right) \times\left(\dot{\boldsymbol{\xi}}^{(k)}-\nabla \phi\right)\right] \quad(k=1,2)
\end{aligned}
$$

Note that in order to calculate the forces in Eq. [9], what is actually needed is the integration $\int_{S_{B}} \phi_{t} n_{i} \mathrm{~d} S$, rather than $\phi_{t}$ itself. The whole term can be immediately obtained from Eqs. 224) and 225, given the body accelerations, which however are unknown before the hydrodynamic forces can be computed. Now let us consider the motion equation for freely moving Body 1 with respect to its center of gravity

$$
\mathbf{M}_{1} \mathbf{A}_{1}=\mathbf{F}_{1}
$$


where $\mathbf{M}_{\mathbf{1}}$ is the mass matrix of Body 1 and $\mathbf{A}_{\mathbf{1}}$ is its acceleration in six degrees of freedom, and the hydrodynamic force on Body 1 is

$$
F_{i}^{(1)}=-\rho \int_{S_{B 1}} \phi_{t} n_{1, i} \mathrm{~d} S-\rho \int_{S_{B 1}}\left(g z+\frac{1}{2} \nabla \phi \cdot \nabla \phi\right) \cdot n_{1, i} \mathrm{~d} S
$$

Substituting Eqs. 24) and (35) into the motion equation Eq. (34) for Body 1 leads to

$$
\begin{array}{r}
{\left[M_{i j}^{(1)}+\rho C_{i j}^{(1)}\right] \cdot A_{i}^{(1)}+\rho D_{i j}^{(1)} \cdot A_{i}^{(2)}=-\rho \Gamma_{i}^{B 1}-\rho \Gamma_{i}^{F}-\rho E_{i}^{(1)}-\rho E_{i}^{(2)}} \\
(i, j=1,2, \cdots, 6)
\end{array}
$$

where

$$
\Gamma_{i}^{B 1}=\int_{S_{B 1}}\left(g z+\frac{1}{2} \nabla \phi \cdot \nabla \phi\right) \cdot n_{1, i} \mathrm{~d} S .
$$

Eq. (36) can be written in the matrix form as

$$
\left[\mathbf{M}_{1}+\mathbf{C}_{1}\right] \mathbf{A}_{1}+\mathbf{D}_{1} \mathbf{A}_{2}=\mathbf{Q}_{1}
$$

Comparing with the motion equation for a single body case, the additional term $\mathbf{D}_{\mathbf{1}} \mathbf{A}_{\mathbf{2}}$ appears, which is apparently contributed by the presence of Body 2. Therefore, in this circumstance the acceleration of Body 1 can not be individually solved.

A similar motion equation for Body 2 can be obtained

$$
\left[\mathbf{M}_{2}+\mathbf{C}_{2}\right] \mathbf{A}_{2}+\mathbf{D}_{2} \mathbf{A}_{1}=\mathbf{Q}_{2}
$$

The appearing term $\mathbf{D}_{\mathbf{2}} \mathbf{A}_{\mathbf{1}}$ is due to the presence of Body 1. Combining these two motion equations (38) and $[39$, we obtain the following 12 linear equations in the matrix form

$$
\left[\begin{array}{cc}
\mathrm{M}_{1}+\mathrm{C}_{1} & \mathrm{D}_{1} \\
\mathrm{D}_{2} & \mathrm{M}_{2}+\mathrm{C}_{2}
\end{array}\right]\left[\begin{array}{l}
\mathrm{A}_{1} \\
\mathrm{~A}_{2}
\end{array}\right]=\left[\begin{array}{l}
\mathrm{Q}_{1} \\
\mathrm{Q}_{2}
\end{array}\right] .
$$

In this way, the accelerations of Body 1 and Body 2 are solved at each time step, and the hydrodynamic forces are computed by substituting back the accelerations as

$$
\begin{aligned}
& \mathbf{F}_{1}=\mathbf{M}_{1} \mathbf{A}_{1}=\mathbf{Q}_{1}-\mathbf{C}_{1} \mathbf{A}_{1}-\mathrm{D}_{1} \mathbf{A}_{2} \\
& \mathbf{F}_{2}=\mathbf{M}_{2} \mathbf{A}_{2}=\mathbf{Q}_{2}-\mathbf{C}_{2} \mathbf{A}_{2}-\mathbf{D}_{2} \mathbf{A}_{1}
\end{aligned}
$$

\section{Constraint matrix approach}

It may be necessary, in some circumstances, to interconnect the adjacent floating bodies, for instance two vessels upon side-by-side operations. With connections, the bodies are not freely moving but constrained by the connections. The additional unknown constraint forces $\mathbf{F}_{\mathbf{c s t}}$ at the connections appear. In order to 
incorporate the constraint forces, the motion equations $(38)$ and $(39)$ need to be modified. The modified motion equations now read

$$
\begin{aligned}
& {\left[\mathbf{M}_{1}+\mathbf{C}_{1}\right] \mathbf{A}_{1}+\mathbf{D}_{1} \mathbf{A}_{2}=\mathbf{Q}_{1}+\mathbf{F}_{\mathbf{e} 1}} \\
& {\left[\mathbf{M}_{2}+\mathbf{C}_{2}\right] \mathbf{A}_{2}+\mathbf{D}_{\mathbf{2}} \mathbf{A}_{1}=\mathbf{Q}_{2}+\mathbf{F}_{\mathrm{e} 2}}
\end{aligned}
$$

where $\mathbf{F}_{\mathbf{e} 1}, \mathbf{F}_{\mathbf{e} 2}$ are the additional forces acting at the gravity centers of the bodies, due to the constraint. A relationship between $\mathbf{F}_{\mathbf{e} 1} / \mathbf{F}_{\mathbf{e} 2}$ and the constraint forces $\mathbf{F}_{\mathbf{c s t}}$ has to be constructed. In the schematic diagram in Fig. 2 (top view), two bodies centered at $\mathbf{X}_{\mathbf{g} 1}=\left(x_{g 1}, y_{g 1}, z_{g 1}\right)$ and $\mathbf{X}_{\mathbf{g} 2}=\left(x_{g 2}, y_{g 2}, z_{g 2}\right)$ are simply connected by a rigid bar. Considering a reference point $\mathbf{X}_{\mathbf{c}}=\left(x_{c}, y_{c}, z_{c}\right)$ in the connection where the constraint forces are $\mathbf{F}_{\mathbf{c s t}}$, the following relations can be formulated

$$
\mathbf{F}_{\mathrm{e} 1}=\mathbf{K}_{1}^{\mathrm{T}} \mathbf{F}_{\text {cst }}, \quad \mathbf{F}_{\mathrm{e} 2}=-\mathbf{K}_{2}^{\mathrm{T}} \mathbf{F}_{\text {cst }}
$$

where $\mathbf{K}_{\mathbf{1}}, \mathbf{K}_{\mathbf{2}}$ are defined as constraint matrices, depending on the connection type and the configuration of the two bodies. Specifically, the constraint matrices mathematically transfer the motions and forces at the gravity centers $\mathbf{X}_{\mathbf{g} 1} / \mathbf{X}_{\mathbf{g} 2}$ to the connection reference point $\mathbf{X}_{\mathbf{c}}$, or vice versa. This is necessary because the constraint forces are assumed to be acting at a specific connection point while the motion equations are formulated with respect to the center of gravity of each body.

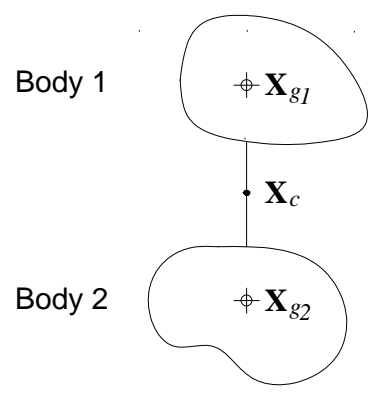

Figure 2: Sketch of two interconnected bodies

Additional equations are required to close the problem, which are naturally the constraint conditions. We take the configuration in Fig. 2 as an example for the purpose of illustration. We consider the displacement $\boldsymbol{\xi}_{c}$ at a particular point $\mathbf{X}_{\mathbf{c}}$ in the connection. With the motions of Body 1, i.e. the displacement $\boldsymbol{\xi}_{1}$ at the gravity center of Body 1 , we can compute the displacement at the point $\mathbf{X}_{\mathbf{c}}$ as $\boldsymbol{\xi}_{\boldsymbol{c}}=\mathbf{K}_{\mathbf{1}} \boldsymbol{\xi}_{\mathbf{1}}$. At the same time, the displacement at the point $\mathbf{X}_{\mathbf{c}}$ can be also computed based on the motions of Body 2 as $\boldsymbol{\xi}_{\mathbf{c}}=\mathbf{K}_{\mathbf{2}} \boldsymbol{\xi}_{\mathbf{2}}$. Therefore, the connection gives the following constraint condition

$$
\mathrm{K}_{1} \xi_{1}=\mathrm{K}_{2} \xi_{2}
$$

The constraint matrices $\mathbf{K}_{\mathbf{1}}, \mathbf{K}_{\mathbf{2}}$ only transfer the displacements at the center of gravity of each body to the connection point of interest. Assume the body displacements are continuous, taking differentiation twice on 
both sides of Eq. (46) leads to

$$
K_{1} A_{1}-K_{2} A_{2}=0
$$

By combining Eqs. (43), 444, 45) and (47), we finally obtain

$$
\left[\begin{array}{ccc}
\mathrm{M}_{1}+\mathrm{C}_{1} & \mathrm{D}_{1} & -\mathrm{K}_{1}^{\mathrm{T}} \\
\mathrm{D}_{2} & \mathrm{M}_{2}+\mathrm{C}_{2} & \mathrm{~K}_{2}^{\mathrm{T}} \\
-\mathrm{K}_{1} & \mathrm{~K}_{2} & \mathbf{0}
\end{array}\right]\left[\begin{array}{c}
\mathrm{A}_{1} \\
\mathbf{A}_{2} \\
\mathrm{~F}_{\text {cst }}
\end{array}\right]=\left[\begin{array}{c}
\mathrm{Q}_{1} \\
\mathrm{Q}_{2} \\
0
\end{array}\right]
$$

The above coupled motion equation system solves the body accelerations and constraint forces at the same time. It has to be made clear that, the constraint matrices generically are 6 by 6 in dimensions such that Eq. 48 is an $18 \times 18$ system. In practice, some degrees of freedom may be not constrained in certain connection types. In these circumstances, we obtain a reduced equation system.

To demonstrate the determination of the constraint matrices, we take two rigidly interconnected bodies as an example, shown in Fig. 2. The two bodies are connected through a horizontal bar. In this simplified configuration, the horizontal rigid bar connects the bodies through their gravity centers $\mathbf{X}_{\mathbf{g} \mathbf{1}}, \mathbf{X}_{\mathbf{g} \mathbf{2}}$. We consider the displacements in 6 DOFs at the middle point of the bar $\mathbf{X}_{\mathbf{c}}$, and the following conditions for the displacement are obtained

$$
\begin{aligned}
\xi_{x}^{(1)}+\left(y_{g 1}-y_{c}\right) \alpha_{z}^{(1)} & =\xi_{x}^{(2)}+\left(y_{g 2}-y_{c}\right) \alpha_{z}^{(2)} \\
\xi_{y}^{(1)} & =\xi_{y}^{(2)} \\
\xi_{z}^{(1)}-\left(y_{g 1}-y_{c}\right) \alpha_{x}^{(1)} & =\xi_{z}^{(2)}+\left(y_{g 2}-y_{c}\right) \alpha_{x}^{(2)} \\
\alpha_{x}^{(1)} & =\alpha_{x}^{(2)} \\
\alpha_{y}^{(1)} & =\alpha_{y}^{(2)} \\
\alpha_{z}^{(1)} & =\alpha_{z}^{(2)}
\end{aligned}
$$

They represent the constraint conditions in Eq. 46. Consequently the corresponding constraint matrices are

$$
\mathbf{K}_{1}=\left[\begin{array}{cccccc}
1 & 0 & 0 & 0 & 0 & \left(y_{g 1}-y_{c}\right) \\
0 & 1 & 0 & 0 & 0 & 0 \\
0 & 0 & 1 & -\left(y_{g 1}-y_{c}\right) & 0 & 0 \\
0 & 0 & 0 & 1 & 0 & 0 \\
0 & 0 & 0 & 0 & 1 & 0 \\
0 & 0 & 0 & 0 & 0 & 1
\end{array}\right] ; \mathbf{K}_{2}=\left[\begin{array}{cccccc}
1 & 0 & 0 & 0 & 0 & \left(y_{g 2}-y_{c}\right) \\
0 & 1 & 0 & 0 & 0 & 0 \\
0 & 0 & 1 & \left(y_{g 2}-y_{c}\right) & 0 & 0 \\
0 & 0 & 0 & 1 & 0 & 0 \\
0 & 0 & 0 & 0 & 1 & 0 \\
0 & 0 & 0 & 0 & 0 & 1
\end{array}\right]
$$

Note that the motion equations are formulated based on the gravitational centers of the bodies, it can be 
further illustrated that the external forces $\mathbf{F}_{\mathbf{e}}$ on the gravitational centers due to the constraint forces are

$$
\begin{aligned}
\mathbf{F}_{\mathbf{e} 1} & =\left[\begin{array}{cccccc}
1 & 0 & 0 & 0 & 0 & 0 \\
0 & 1 & 0 & 0 & 0 & 0 \\
0 & 0 & 1 & 0 & 0 & 0 \\
0 & 0 & -\left(y_{g 1}-y_{c}\right) & 1 & 0 & 0 \\
0 & 0 & 0 & 0 & 1 & 0 \\
\left(y_{g 1}-y_{c}\right) & 0 & 0 & 0 & 0 & 1
\end{array}\right] \cdot \mathbf{F}_{\mathbf{c s t}}=\mathbf{K}_{1}^{\mathbf{T}} \mathbf{F}_{\mathbf{c s t}} \\
\mathbf{F}_{\mathbf{e} 2} & =\left[\begin{array}{cccccc}
1 & 0 & 0 & 0 & 0 & 0 \\
0 & 1 & 0 & 0 & 0 & 0 \\
0 & 0 & 1 & 0 & 0 & 0 \\
0 & 0 & \left(y_{g 2}-y_{c}\right) & 1 & 0 & 0 \\
0 & 0 & 0 & 0 & 1 & 0 \\
\left(y_{g 2}-y_{c}\right) & 0 & 0 & 0 & 0 & 1
\end{array}\right] \cdot\left(-\mathbf{F}_{\mathbf{c s t}}\right)=-\mathbf{K}_{2}^{\mathbf{T}} \mathbf{F}_{\mathbf{c s t}}
\end{aligned}
$$

The constraint matrix can be constructed the same way for other connection types. Details are presented in Section 6 .

\section{Two freely moving barges}

To verify the approach of coupled auxiliary functions presented in Section 3 , we first consider two freely floating barges asymmetrically arranged with a relatively large spacing between them. The coordinate system and configuration of the two identical barges are shown in Fig. 3(a) The two barges are $2.0 \mathrm{~m}$ long and $1.0 \mathrm{~m}$ wide, and have a draft of $0.3 \mathrm{~m}$. They are separated by a distance of $1 \mathrm{~m}$. The center of gravity of each barge is assumed to locate coincided with its center of buoyancy in still water, i.e. $z_{g}=-0.15 \mathrm{~m}$. The local coordinate system $O^{\prime} x^{\prime} y^{\prime} z^{\prime}$ is set at the center of gravity of each body and initially parallel to the global coordinate system $O x y z$. In the following content, the subscript notations $x, y, z$ indicate the motion directions in the global coordinate system while surge, sway, heave, roll, pitch and yaw are corresponding to barges motions in their respective local coordinate systems. The water depth in the circular tank is $3 \mathrm{~m}$, which is considered as a deep water. The tank radius is either 2.5 times the barge length or four times the incident wave length, whichever is larger. A mesh on the free surface and the barges is shown in Fig. 3(b)] It has to be stated that this configuration does rarely appear in reality, with one barge perpendicular to another barge in close proximity. In marine offshore engineering, two vessels staying close to each other are normally either in tandem or side-by-side configuration. For the purpose of illustration, we investigate this imaginary complicated case which is asymmetric and large-space separated. This is to avoid possible gap resonance (Feng and Bai 2015) which is not of interest here. We focus more on the dynamic responses in the time domain, in order to demonstrate the effectiveness of the proposed coupled auxiliary function approach. 
For the purpose of illustration, we assume a uniform mass distribution in the barges, and the water density is specified as $1025 \mathrm{~kg} / \mathrm{m}^{3}$.

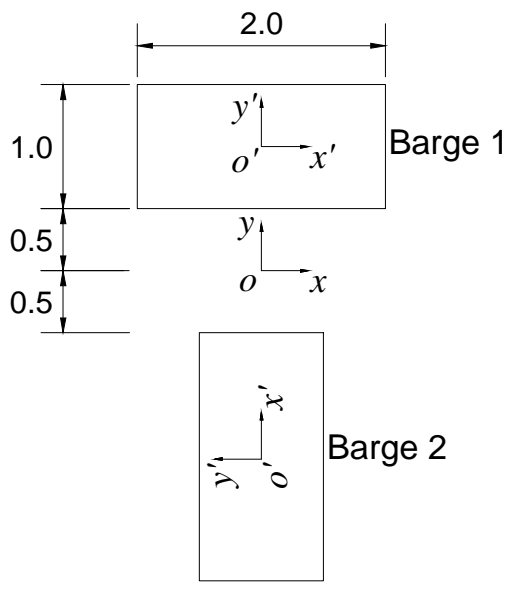

(a)

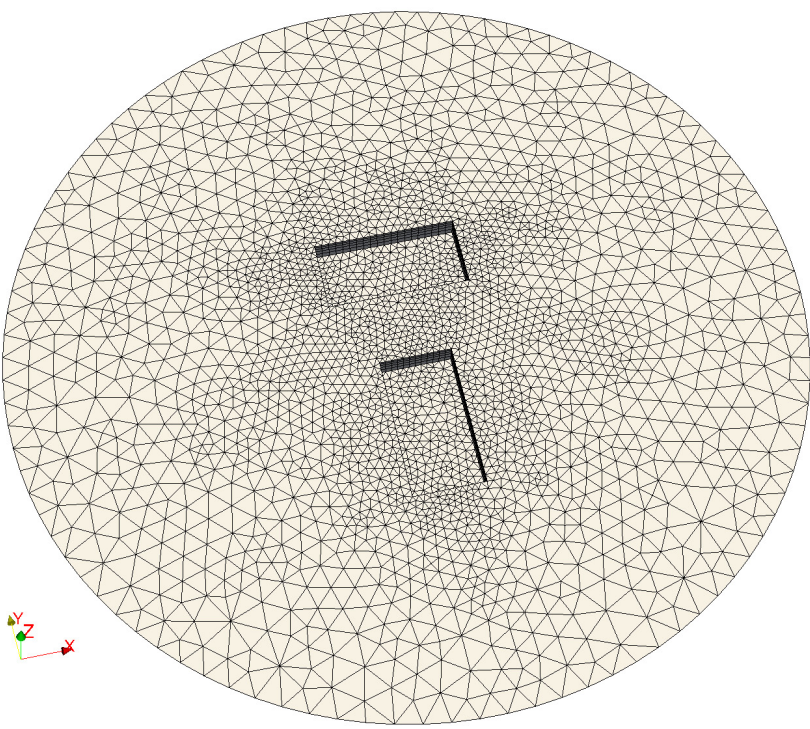

(b)

Figure 3: Two freely floating barges: (a) configuration (unit in ' $m$ ') and (b) mesh on free surface and barges. The local barge motions are defined in the local coordinate system $o^{\prime} x^{\prime} y^{\prime} z^{\prime}$, so that surge, sway and heave displacements are translational motions along the $x^{\prime}-, y^{\prime}$ - and $z^{\prime}$-axis. Roll, pitch and yaw displacements are rotational motions about the $x^{\prime}$-, $y^{\prime}$ - and $z^{\prime}$-axis

\subsection{Results of wave elevation and barge motions}

A $0^{\circ}$ incident wave of frequency $\omega=6.0 \mathrm{rad} / \mathrm{s}$, propagating along the positive $x$ direction, is imposed in the tank. The wave steepness is relatively low with a wave amplitude of $0.005 \mathrm{~m}$, in order to compare with linear results. Simulations essentially run for more than 20 periods of the incident wave to achieve the steady state. Mesh convergence tests are firstly performed to ensure that a proper computational mesh (accurate and less costly) is adopted in the simulations. In this model, the free surface unstructured mesh is generated by an automatic Delaunay triangulation algorithm. Mesh quality and density are controlled by the element size on the solid boundaries as well as the number of nodes inserted on the free surface. The number of inserted nodes has to be carefully selected such that the mesh is smoothly stretched from small local elements near bodies to large ones at the numerical beach. It is the mesh quality on the free surface around the bodies that determines the accuracy of the results. In the response of wave elevation at the center of the tank in Fig. 4, three mesh configurations, Mesh 1, Mesh 2 and Mesh 3 are included. The local element sizes on the free surface surrounding the bodies for Mesh 1, Mesh 2 and Mesh 3 are approximately one twentieth, one thirtieth and one fortieth of the wave length, respectively. Mesh 1 configuration includes 4550 elements and 10469 nodes in the computational domain while 6318 elements and 14509 nodes are contained 
in Mesh 2, 9498 elements and 22881 nodes in Mesh 3. The time histories of the elevations for these three mesh configurations seem almost identical, and only less than $1 \%$ difference is observed at the maxima. Mesh convergence for wave forces is also achieved in Figs. $5(\mathrm{a})$ and 5(b), where the responses of surge force $F_{x}$ and pitch moment $M_{y}$ on Barge 1 in two periods are plotted, respectively. This simple mesh convergence study demonstrates that the computational mesh has converged for the configuration of Mesh 1 . In all the following cases, we adopt a similar mesh density to that in Mesh 1 configuration.

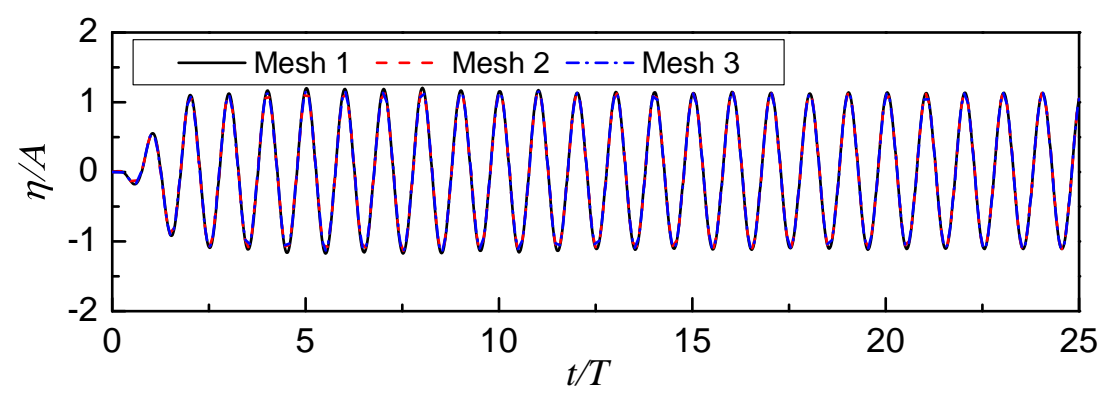

Figure 4: Wave elevation at the center of the free surface for two different meshes

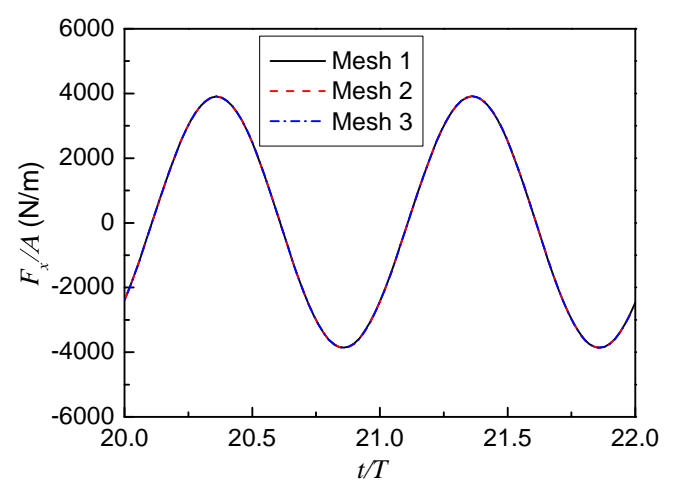

(a)

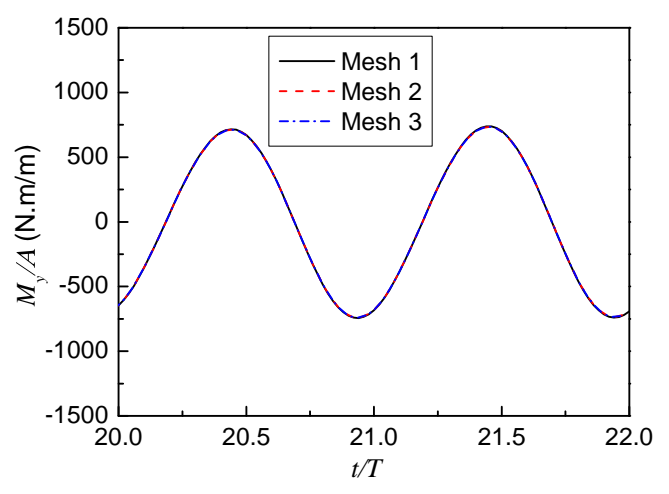

(b)

Figure 5: Wave forces on Barge 1 for two asymmetrically arranged barges subject to a $0^{\circ}$ incident wave of frequency $\omega=6.0 \mathrm{rad} / \mathrm{s}$ : (a) $F_{x}$ and (b) $M_{y}$

We first present the response of wave elevation on the free surface in the tank. Fig. 6 shows the contour map of maximum elevation on the free surface, which is normalized by the incident wave amplitude. The contour map characterizes the nature that the upfront of the barges is subject to higher wave run-up while the leeside area is well sheltered, and this effect is more pronounced for Barge 2 which is in its beam sea. As the two barges are relatively large-spaced, no significant wave trapping in-between is observed. Nevertheless, the wave pattern around each barge is considerably disturbed by the presence of the other, compared to a single barge case.

We focus more on the motion responses of the bodies. Fig. 7 plots typical motion responses in 6 DOFs of Barge 1. The motions are normalized by the incident wave amplitude. It is clear that the motions of surge, 


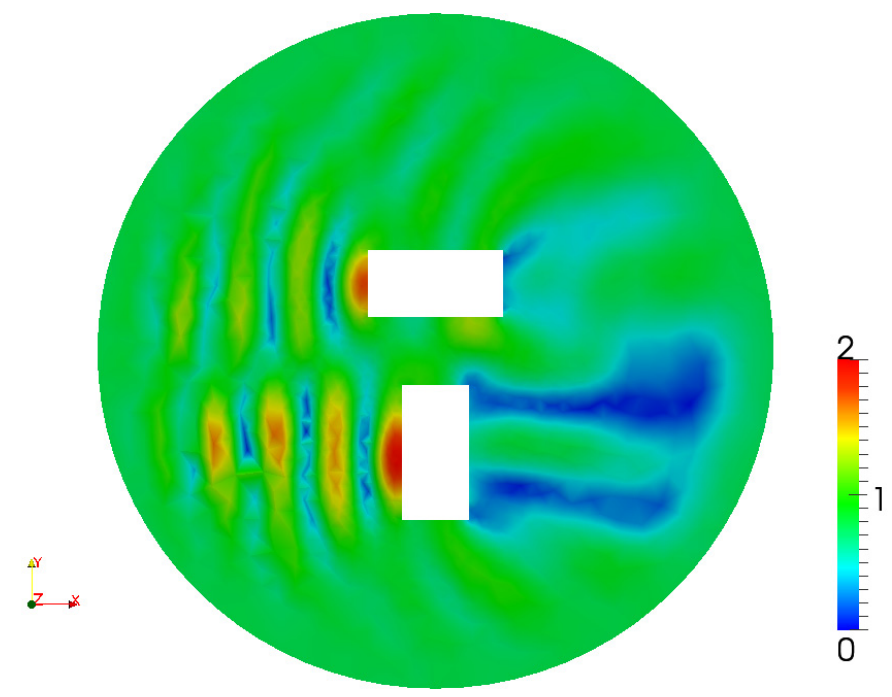

Figure 6: Wave amplitude for two asymmetrically arranged barges subject to a $0^{\circ}$ incident wave of frequency $\omega=$ $6.0 \mathrm{rad} / \mathrm{s}$

sway and yaw demonstrate a very different character from those of heave, roll and pitch. The increasing mean surge motion in Fig. 7(a) shows that Barge 1 is drifting downstream in the direction of the incident wave; at the same time Barge 1 is slightly separated from Barge 2 with a positive sway drift, as can be seen in Fig. 7(b), Unlike the slow drift, motions of heave, roll and pitch oscillate around zero. This is due to the fact that no mooring system is implemented in the model and the second-order mean forces in the directions of surge, sway and yaw result in a drift effect; whereas hydrostatic restoring forces/moments balance the motions of heave, roll and pitch.

A noteworthy feature in the motions of heave, roll and pitch is that they demonstrate a relatively long transient response (some 15 periods) and the transient amplitude could be higher than the steady-state response. Nonetheless, their steady-state responses are still single-harmonic dominant, since the incident wave is almost linear.

Apart from the motion of each barge itself, the relative motion may also be of great importance, which is crucial for side-by-side operations. Fig. 8 shows the relative motions of Barge 2 to Barge 1 in the $x, y, z$ directions. It is found that Barge 2 drifts downstream faster than Barge 1; this is because drift force in the $x$ direction on Barge 2 is greater than that on Barge 1 as the wave is in the beam sea of Barge 2. The negative relative motion (the harmonic component is quite low) in the $y$ direction again reveals that the $0^{\circ}$ incident wave is separating Barges 1 and 2 at the frequency $\omega=6.0 \mathrm{rad} / \mathrm{s}$, and this is mainly due to the anti-directional drift forces on the two barges. Interestingly, the maximum relative heave is larger than the heave of each barge as their heaves have a phase difference. This suggests that the possibly enlarged relative motions must be taken into consideration while performing side-by-side operations.

The velocity and acceleration in every DOF of each barge can be plotted as well. We only present the corresponding responses in surge and pitch of Barge 1 in Fig.9(a) and 9(b), which represent typical responses 


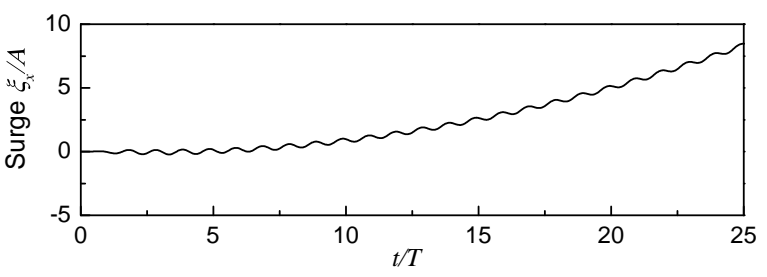

(a) Surge

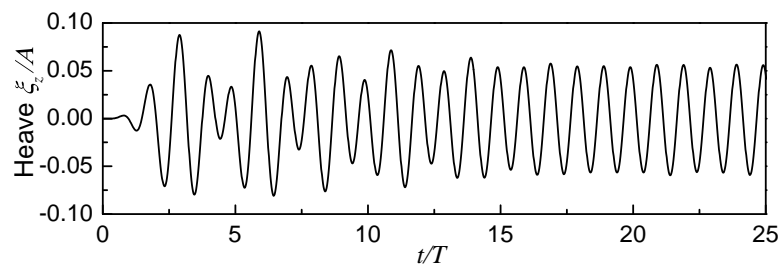

(c) Heave

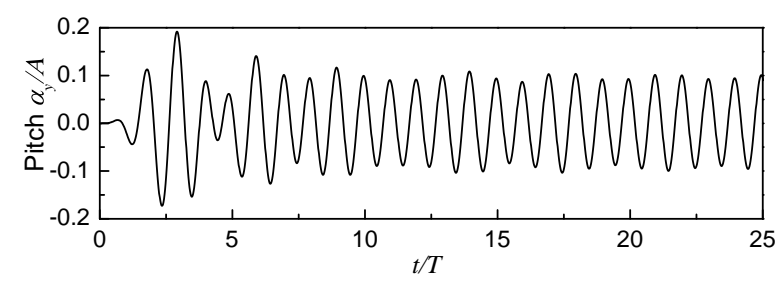

(e) Pitch

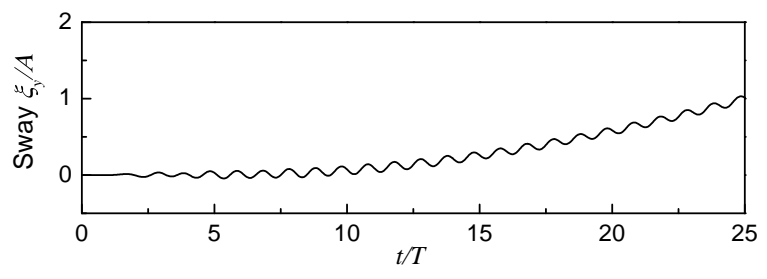

(b) Sway

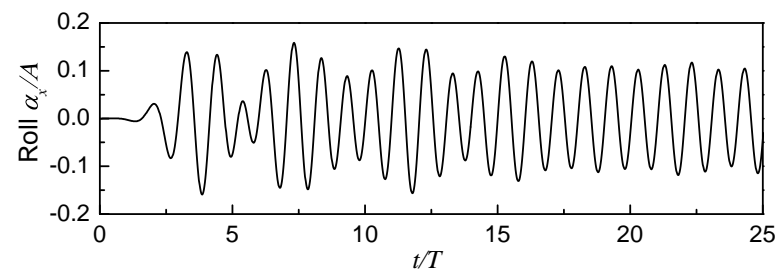

(d) Roll

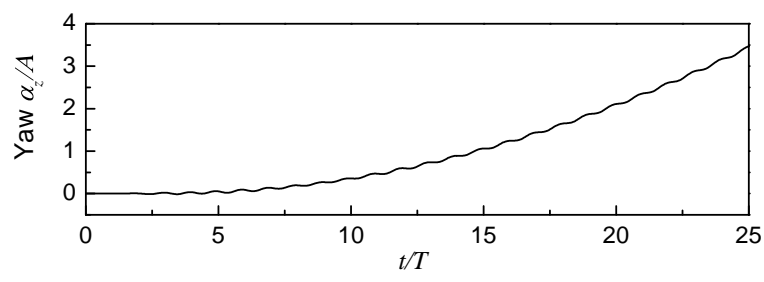

(f) Yaw

Figure 7: Motions of Barge 1 for two asymmetrically arranged barges subject to a $0^{\circ}$ incident wave of frequency $\omega=6.0 \mathrm{rad} / \mathrm{s}$

with and without drift effect, respectively. The top panel plots the displacement, middle panel the velocity and bottom panel the acceleration. The acceleration in surge displays a single harmonic response, but its mean value is not zero. This non-zero mean acceleration leads to a linear increase of the mean velocity as time evolves. It can be easily illustrated by time integration of the acceleration. Then a second timeintegration shows a quadratic component in the displacement response, as shown in the surge time history. Nevertheless, the mean surge acceleration is generally quite small in this mild wave case, such that the drift effect is not that significant. Unlike the surge, the pitch acceleration has a zero mean, resulting in harmonic response in the pitch displacement.

\subsection{Harmonic analysis}

The results from fully nonlinear simulations essentially include all features of nonlinearity. It could be of interest to explore the harmonics other than the incident wave frequency dominated component. A harmonic analysis can be carried out using the FFT (Fast Fourier Transform) technique. For any time history of interest, we select the steady-state response of 10-period duration and transfer the time series into a frequency-domain response, such that harmonic component at each frequency (within frequency resolution) can be obtained. 


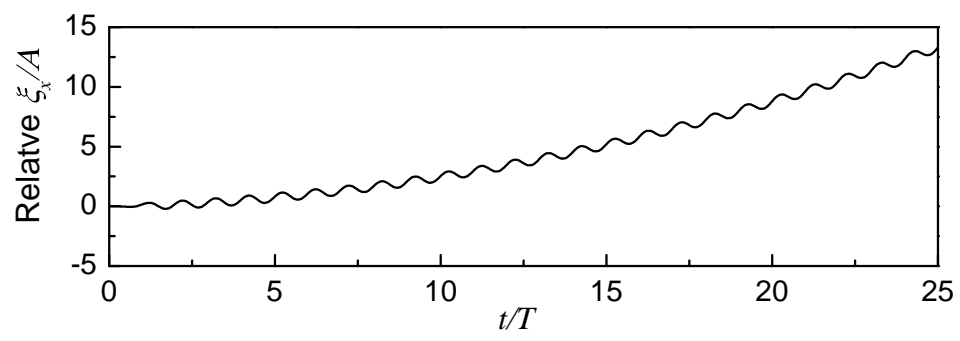

(a)

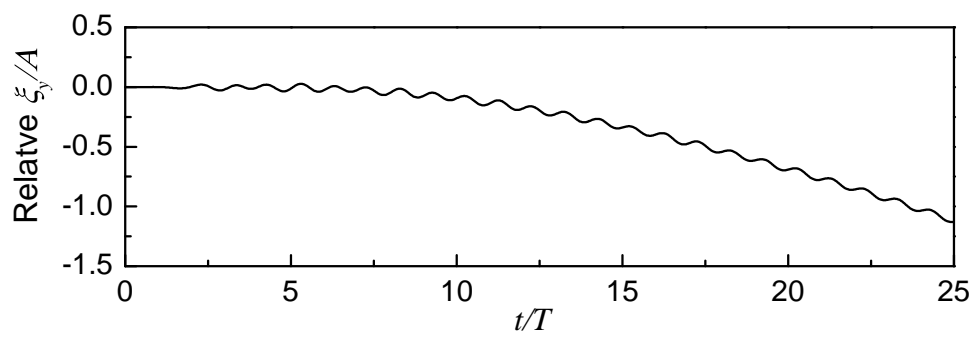

(b)

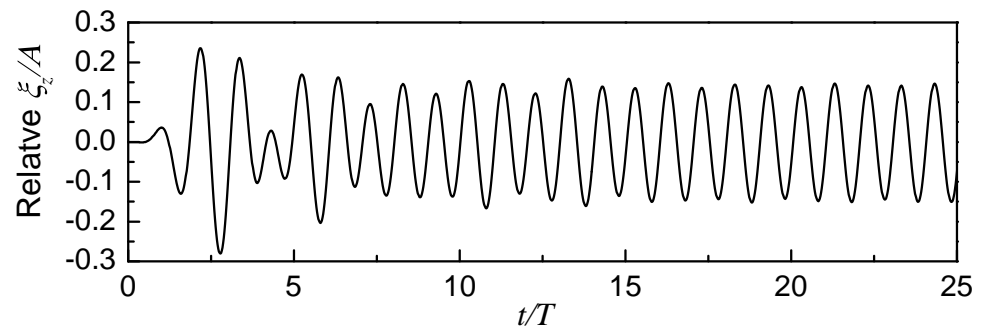

(c)

Figure 8: Relative motions of Barge 2 to Barge 1 for two asymmetrically arranged barges subject to a $0^{\circ}$ incident wave of frequency $\omega=6.0 \mathrm{rad} / \mathrm{s}$ : (a) in $x$ direction; (b) in $y$ direction and (c) in $z$ direction

With the motion responses of heave, roll and pitch (no drift effect), we can directly perform the FFT to obtain their response spectra. As for the responses of surge, sway and yaw where the drift effect is significant, the FFT can not be directly applied on the time histories. Before performing spectrum analysis, we need to eliminate the drift effect or the quadratic component due to the mean acceleration, to recover the single-harmonic dominated response. To do this, we assume the response of acceleration in a certain DOF has a form

$$
a(t)=\sum_{i} A_{a i} \cos \left(\omega_{i} t-\varphi_{i}\right)+a_{0}
$$

where $A_{a i}$ and $\varphi_{i}$ are the acceleration amplitude and phase corresponding to the harmonic at $\omega_{i}$, and $a_{0}$ is the mean acceleration. This leads to the velocity as

$$
v(t)=\sum_{i} A_{v i} \sin \left(\omega_{i} t-\varphi_{i}\right)+a_{0} t+v_{0}
$$



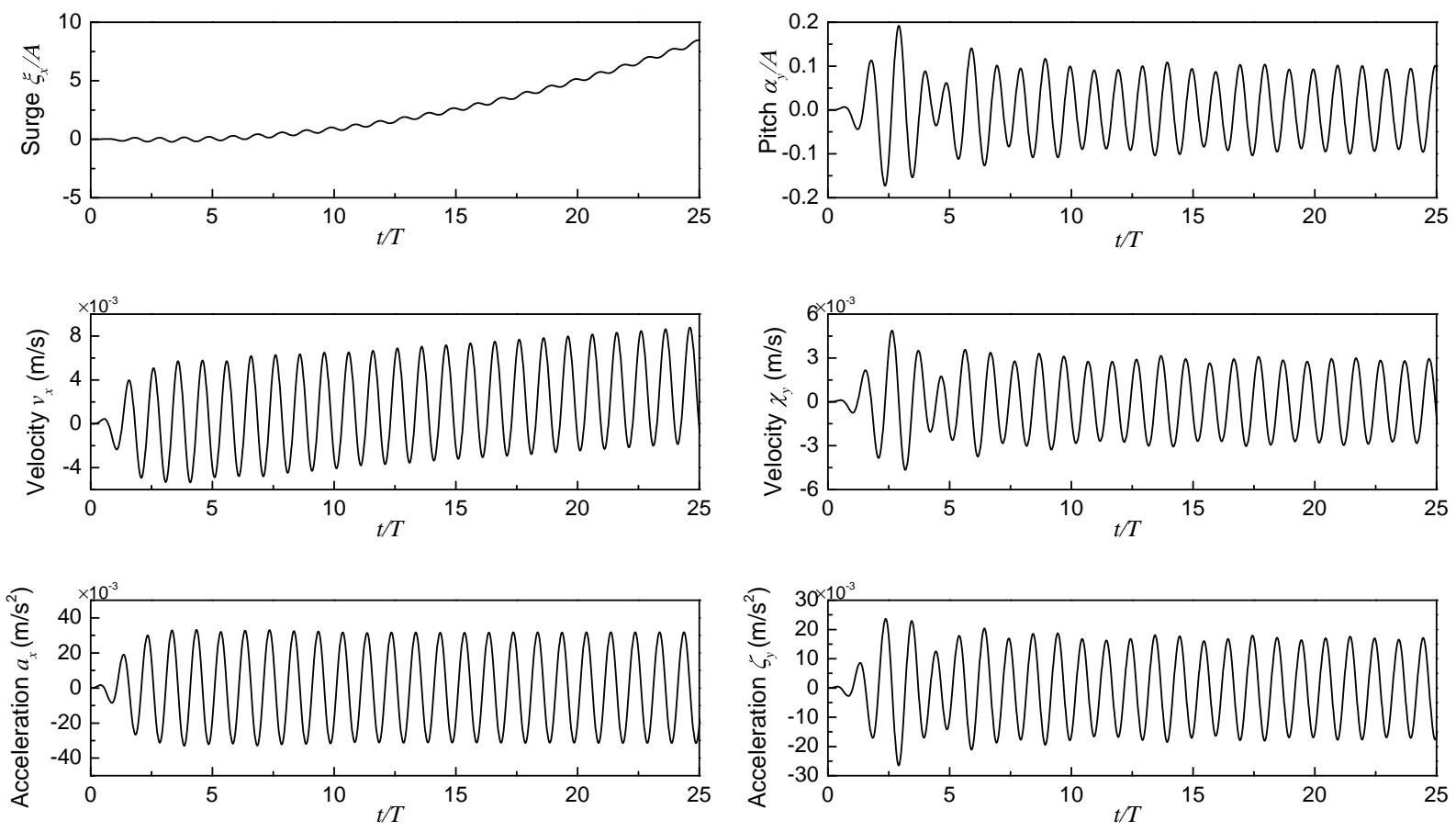

(a)

(b)

Figure 9: Responses of Barge 1 for two asymmetrically arranged barges subject to a $0^{\circ}$ incident wave of frequency $\omega=6.0 \mathrm{rad} / \mathrm{s}$ : (a) Surge and (b) Pitch. Top panel is displacement, middle panel velocity and bottom panel acceleration.

and the displacement as

$$
\xi(t)=\sum_{i} A_{\xi i} \cos \left(\omega_{i} t-\varphi_{i}\right)+\frac{1}{2} a_{0} t^{2}+v_{0} t+\xi_{0}
$$

where $v_{0}$ and $\xi_{0}$ are the mean velocity and displacement, $A_{v i}$ and $A_{\xi i}$ are the amplitudes of velocity and displacement corresponding to the component at $\omega_{i}$.

We process the displacement response $\xi(t)$ by subtracting the components due to the mean velocity $v_{0}$ and the mean acceleration $a_{0}$, i.e., $\left(\frac{1}{2} a_{0} t^{2}+v_{0} t\right)$. After this processing, we are able to obtain the responses of surge, sway and yaw with the drift effect eliminated. Figs. 10(a) and 10(b) show the regenerated responses of surge velocity and displacement. It is now clear to see that both the velocity and displacement demonstrate a single-harmonic dominated response. Harmonic analysis then can be performed based on the regenerated responses. This post-process is utilized in obtaining the first order responses for surge, sway and yaw presented in the next subsection.

\subsection{Validation of motions}

The fully nonlinear potential flow model, based on which the present model is developed, has been extensively validated in the previous work, i.e., Bai and Eatock Taylor (2006), Bai et al. (2014) and Feng and Bai (2015). As a further validation of the present model, especially for the newly derived approach of calculating hydrodynamic responses using the coupled auxiliary functions, we have here compared our 


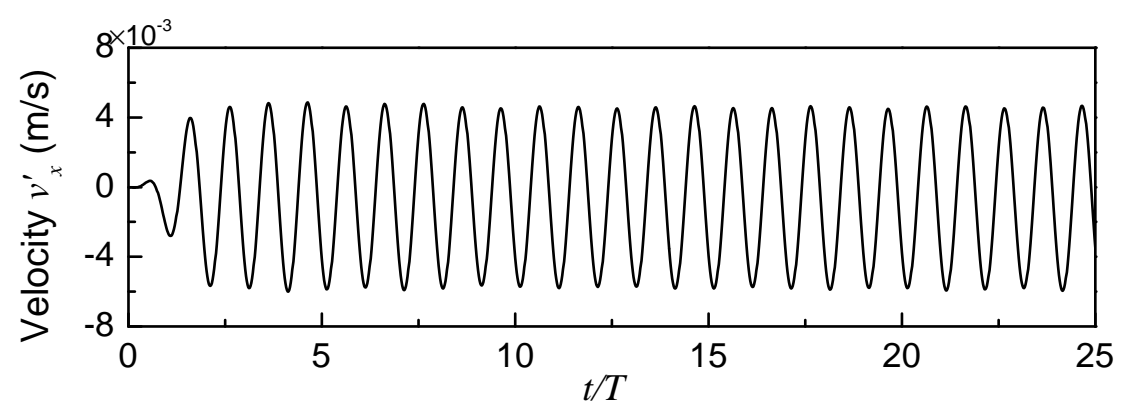

(a)

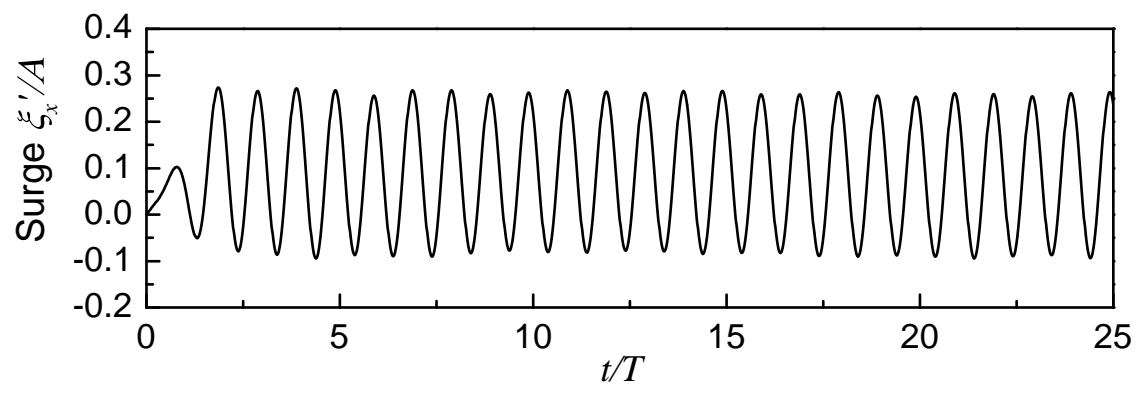

(b)

Figure 10: Regenerated responses of Barge 1 to eliminate the drift effect: (a) Surge velocity and (b) Surge displacement

simulation results with HydroStar ${ }^{\circledR}$, a commercialized linear frequency-domain software package. First order responses are extracted by the FFT from the present fully nonlinear results. Fig. 11 shows the comparisons of the first order motions of Barge 1 between the present model and HydroStar ${ }^{\circledR}$. The RAOs are normalized by the incident wave amplitude. Simulations are performed over the frequency range 5-8 rad/s. The overall agreements for 6 DOF motions are extremely satisfactory. Only very small discrepancy is observed in the yaw response. A possible explanation is that in the present time-domain model the relative position of the two floating bodies can be captured as the slight change in the body position occurs during the simulation, while it remains the same in the frequency-domain software HydroStar ${ }^{\circledR}$ since the Taylor series expansion is used in the method. A noteworthy character for the sway in Fig. 11(b) is that, there apparently appears a peak near the frequency $6.0 \mathrm{rad} / \mathrm{s}$. This is induced by the presence of Barge 2 where a relatively significant wave is nearly trapped between the two barges at this frequency, even though the barges are largely separated. Theoretically, without Barge 2, there should be no sway force on Barge 1 in a $0^{\circ}$ wave. Physically, a mild standing wave is partially trapped between the barges. Given the relatively large separation distance inbetween, the influence of possible resonance is not as significant as the case of a narrow gap, such that the response is not that much amplified as in a gap resonance case.

A similar comparison of first order motions of Barge 2 is presented in Fig. 12. Again, the comparisons in 6 DOFs are agreeable. The good agreement of the body motions well validates the newly proposed approach for solving the coupled motion equations. We then have to emphasize that the value of the present nonlinear 
time-domain simulation attributes to its feature of capturing higher- or lower-frequency components, which cannot be easily obtained from a linear frequency-domain model, saying, HydroStar ${ }^{\circledR}$ and other commercial software used in the industry.

\subsection{Movement trajectory}

It is valuable to plot the trajectory of the barges, in order to gain a direct image of their movements. Fig. 13 presents the acceleration of Barge 1 in the $y$ and $z$ directions versus its horizontal acceleration along the $x$-axis. The trajectories are plotted from $t=10 T$ to $t=30 T$. Clearly both of them have reached steady states (repeating trajectory) after $t=10 T$ showing a periodic near-elliptic locus. This is consistent with the motion responses of the barge. The translational accelerations are single-harmonic dominant such that the locus of a sinusoidal response versus another of a different amplitude results in an ellipse. The shape of the ellipse represents the phase and amplitude difference of motions between these two directions.

\section{Two interconnected moving barges}

After simulating the freely moving barges and validating the coupled auxiliary functions approach, we now investigate two side-by-side interconnected barges. For validation, a simple case considered here is two tandem arranged barges connected by a rigid bar subject to a $0^{\circ}$ wave, as studied in Newman (1994). The configuration is shown in Fig. 14. The two identical barges are $40 \mathrm{~m}$ long, $10 \mathrm{~m}$ beam and $5 \mathrm{~m}$ draft, separated by a $10 \mathrm{~m}$ distance. The barges are assumed to be in static equilibrium with uniform mass distribution throughout the length, beam and depth. The water depth is infinite (we put it at $1000 \mathrm{~m}$ in our numerical model). The two barges are connected end-to-end by a rigid bar. Fig. 15 shows the comparison of vertical motions with the results of Newman (1994), who used a mode expansion method to calculate the motion in his linear frequency-domain model. The solid line shows the vertical motion response at the middle point of the rigid bar by Newman's mode expansion model. Our results of vertical motions of both the upwave and leeside barges are presented and they are essentially very close to those of Newman (1994). Note that there is slight difference in motions between the upwave and leeside barges, and the vertical motion at the middle point of the rigid bar is the averaged vertical responses of the two barges.

Comparisons are also made for a case of two hinged barges of the same configuration (Fig. 14). The hinge axis is located at the origin $O$ in the middle of the rigid connection. The vertical motion of the hinge (not the barge itself) is presented in Fig. 16(a) and the hinge rotation, relative pitch amplitudes of the two barges, normalized by $2 k A$, is compared in Fig. 16(b), The agreements are very good, which well validates the proposed constraint matrix method in modelling the interconnection.

In order to evaluate the effects of the different interconnections, we consider two types of simple connections: rigid connection and middle-hinge connection. As has been explained, a more commonly seen configuration for two close vessels is the side-by-side arrangement for operations. In this regard, we consider 


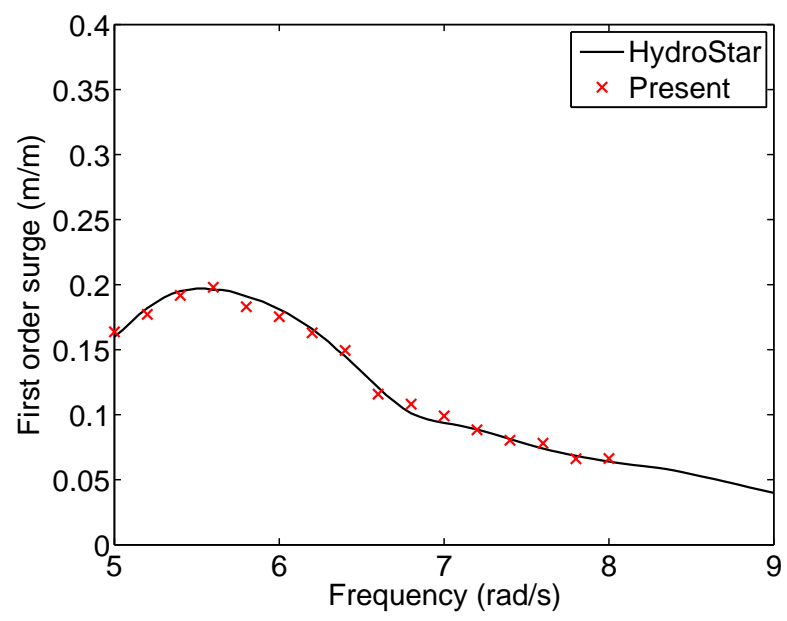

(a)

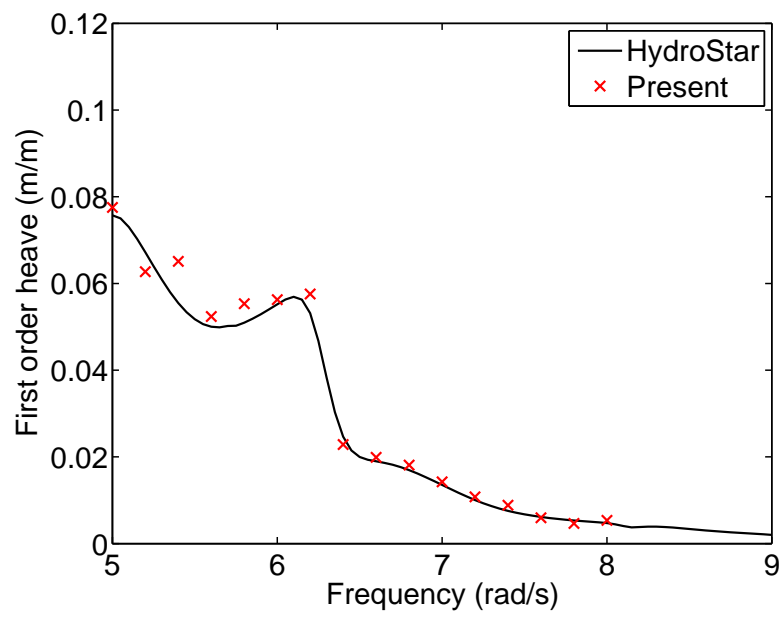

(c)

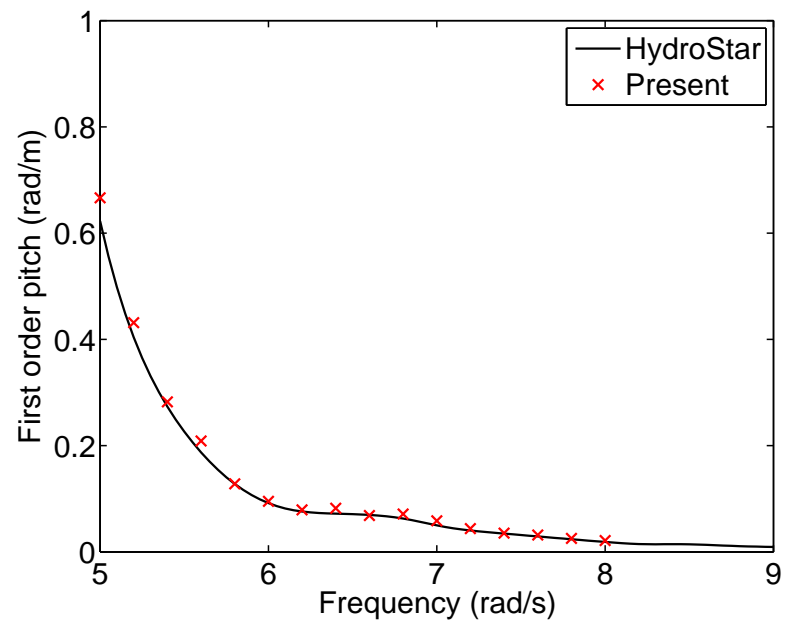

(e)

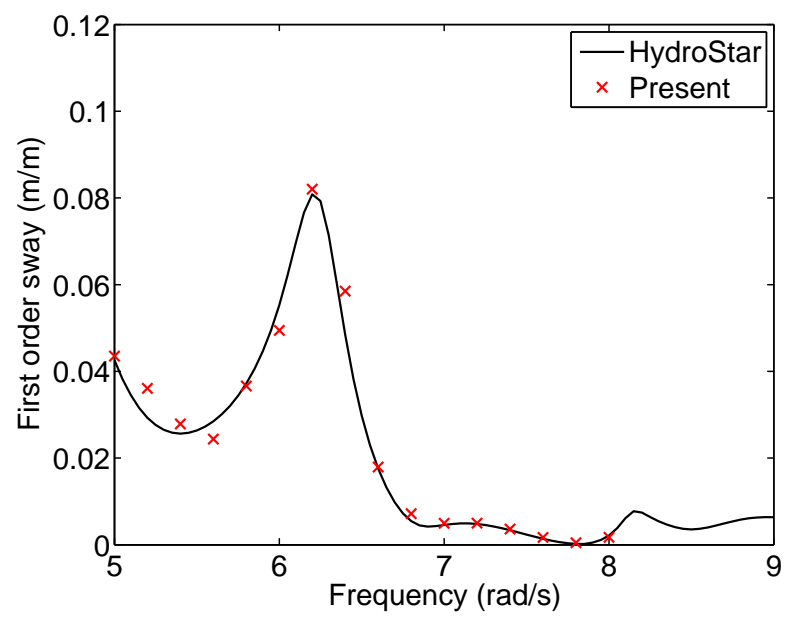

(b)

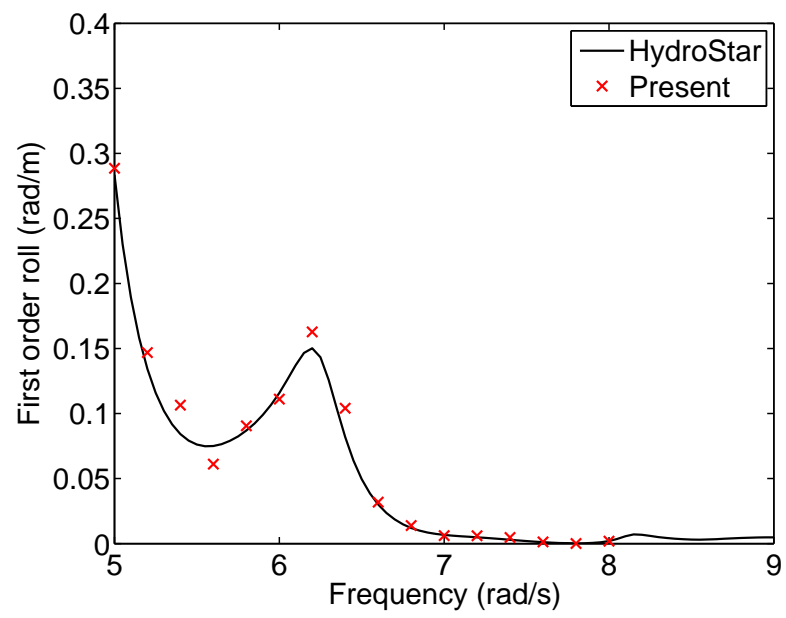

(d)

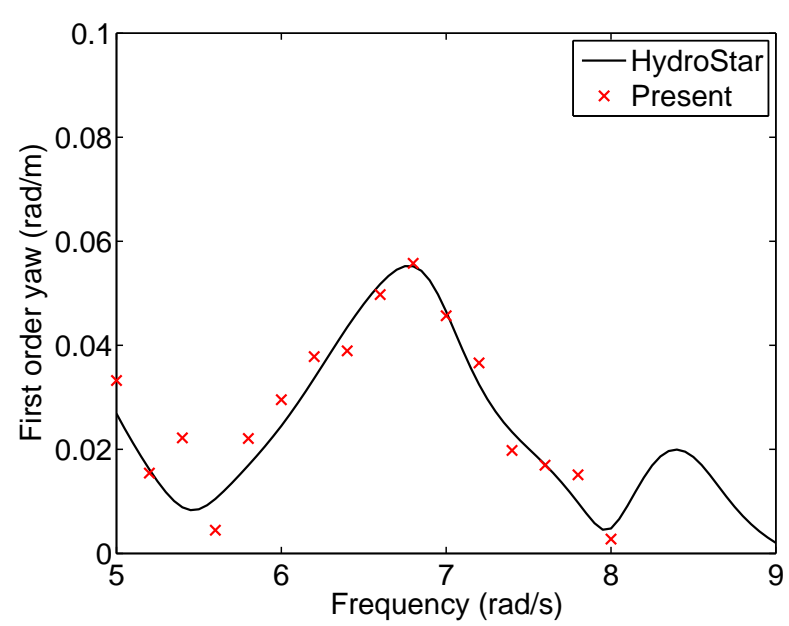

(f)

Figure 11: Comparisons of first order motions of Barge 1 for two asymmetrically arranged barges subject to a $0^{\circ}$ incident wave: (a) $\xi_{x} ;$ (b) $\xi_{y} ;$ (c) $\xi_{z} ;$ (d) $\alpha_{x} ;$ (e) $\alpha_{y}$ and (f) $\alpha_{z}$ 


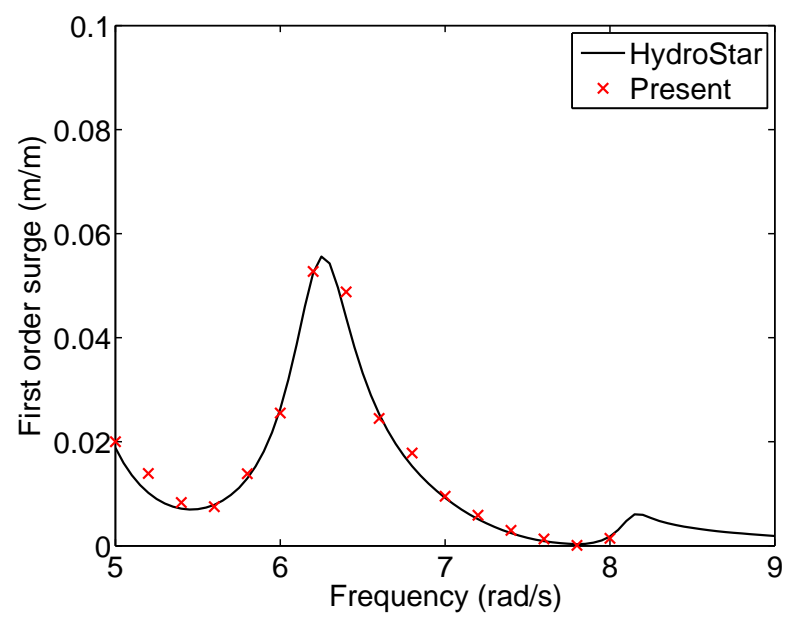

(a)

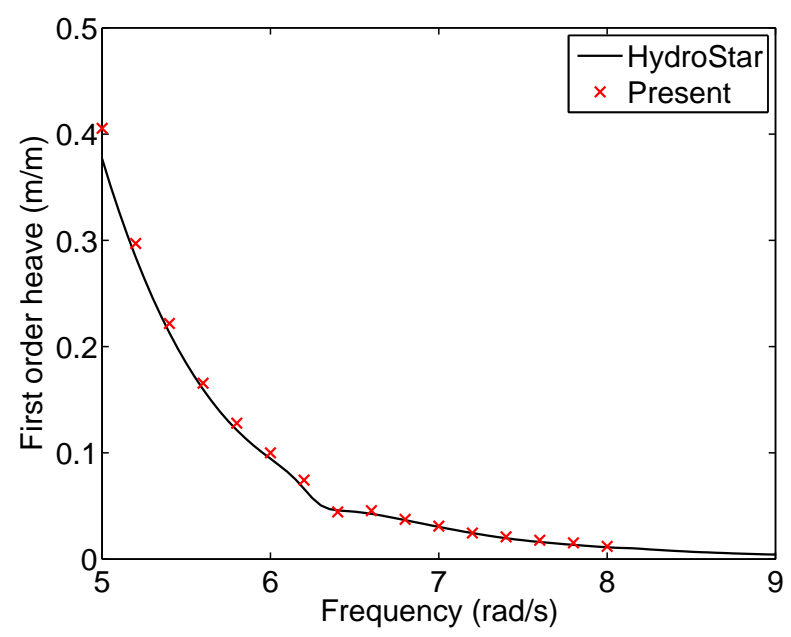

(c)

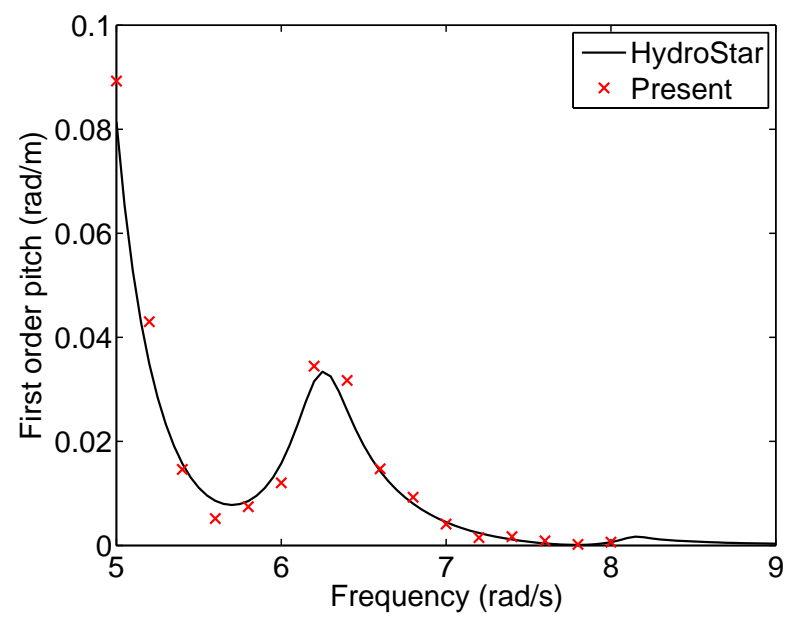

(e)

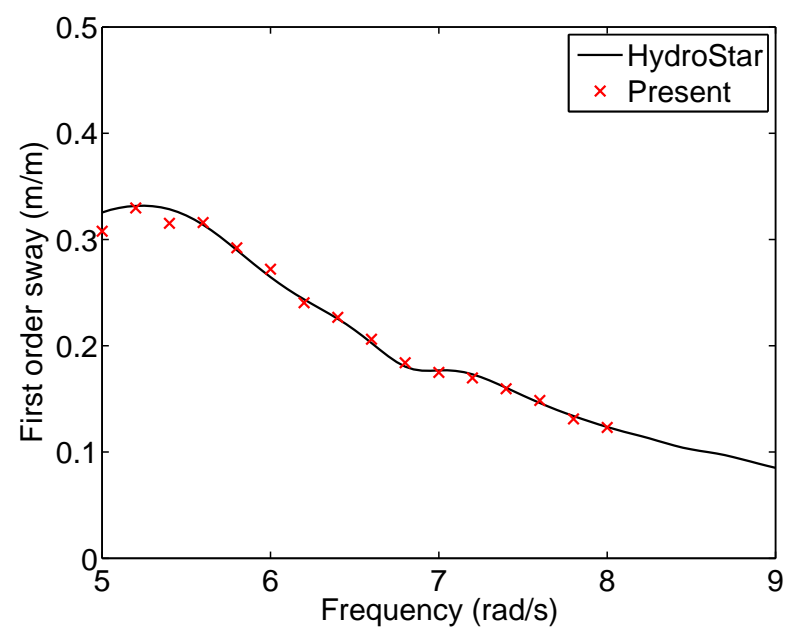

(b)

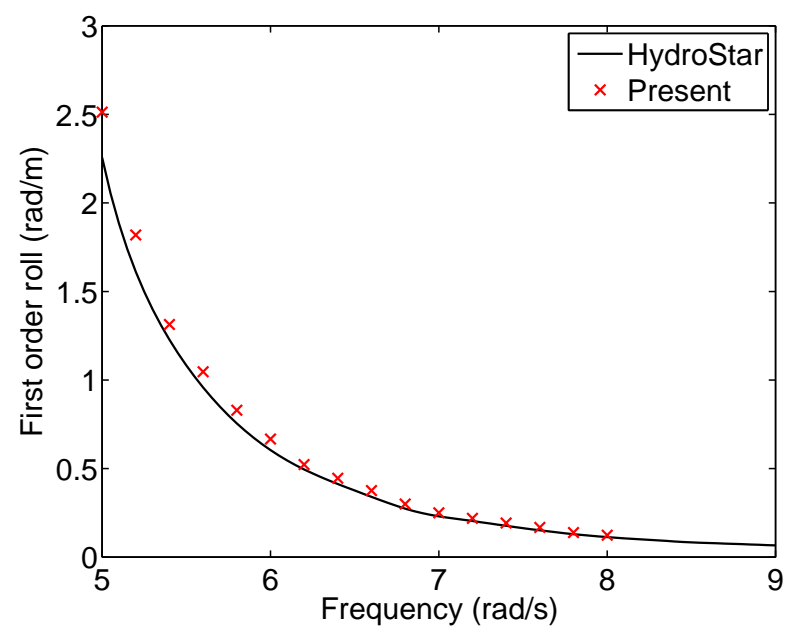

(d)

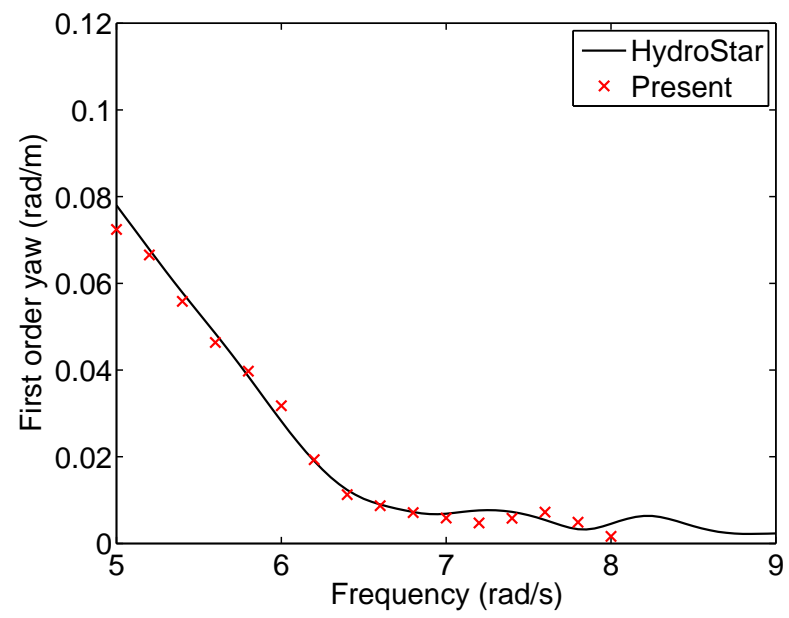

(f)

Figure 12: Comparisons of first order motions of Barge 2 for two asymmetrically arranged barges subject to a $0^{\circ}$ incident wave: (a) $\xi_{y} ;$ (b) $\xi_{x} ;$ (c) $\xi_{z} ;$ (d) $\alpha_{y} ;$ (e) $\alpha_{x}$ and (f) $\alpha_{z}$ 


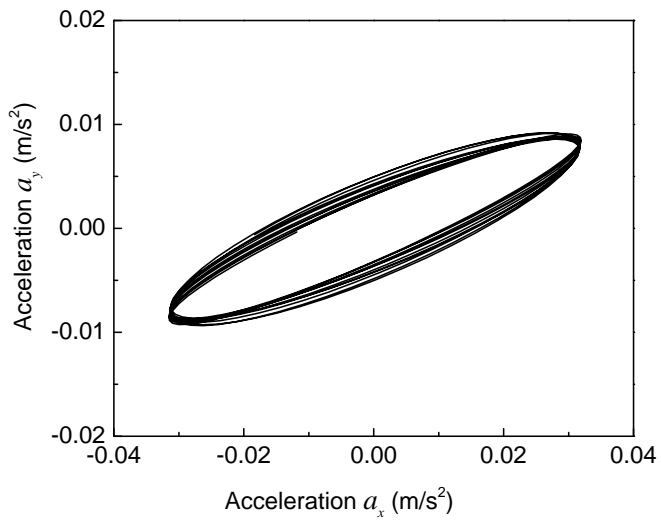

(a)

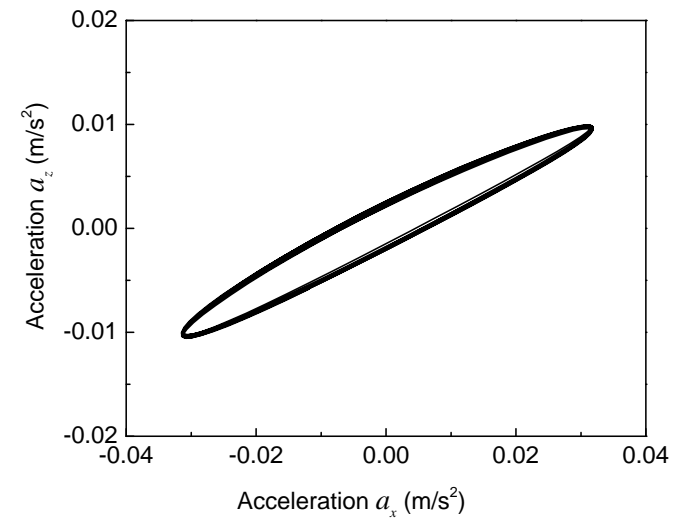

(b)

Figure 13: Trajectories of accelerations of Barge 1 for two asymmetrically arranged barges subject to a $0^{\circ}$ incident wave of frequency $\omega=6.0 \mathrm{rad} / \mathrm{s}$ : (a) $a_{2}$ versus $a_{1}$ and (b) $a_{3}$ versus $a_{1}$

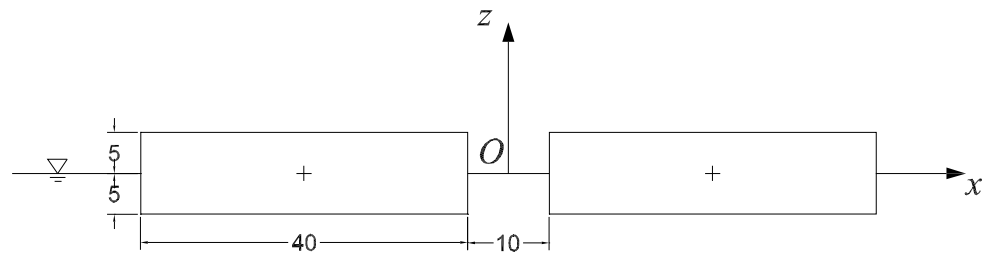

Figure 14: Two rigidly connected barges in tandem as studied in Newman (1994)

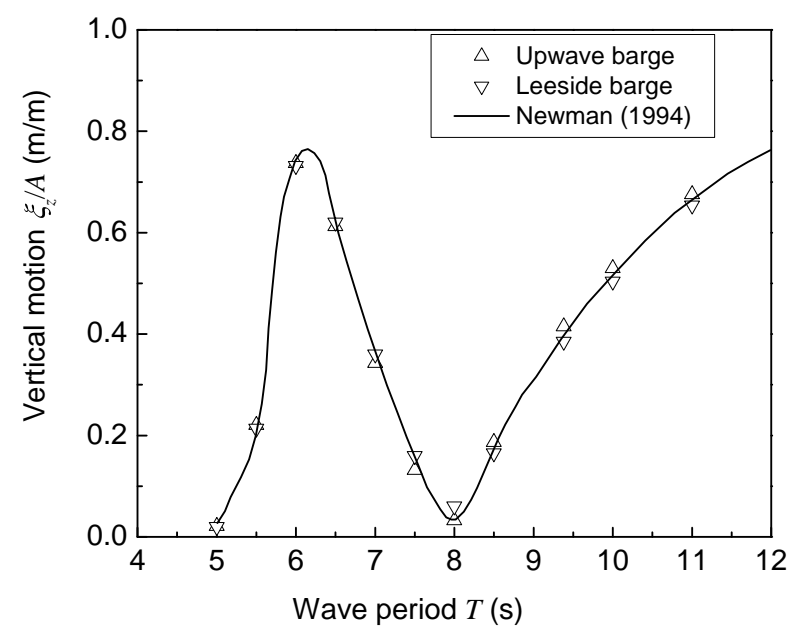

Figure 15: Comparison of vertical motions of rigidly connected barges

two identical rectangular barges, side-by-side arranged, as an example for demonstrating the proposed constraint matrix method for modelling the connections. Plan views of the two types of connections are shown in Fig. 17. Each barge dimension is the same as in Fig. 3(a), which is $2 \mathrm{~m} \times 1 \mathrm{~m}$ with a draft of $0.3 \mathrm{~m}$. The separation distance is $1 \mathrm{~m}$. The origin of the global coordinate system is placed at the middle of the 


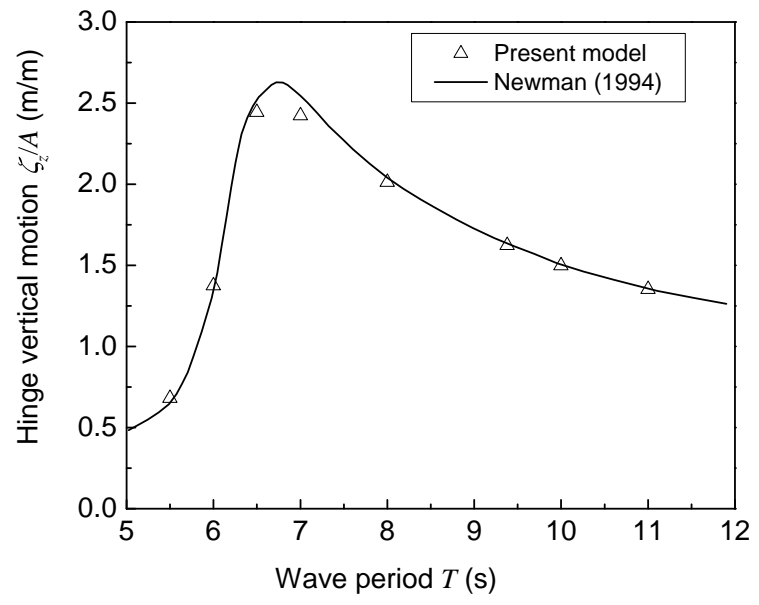

(a)

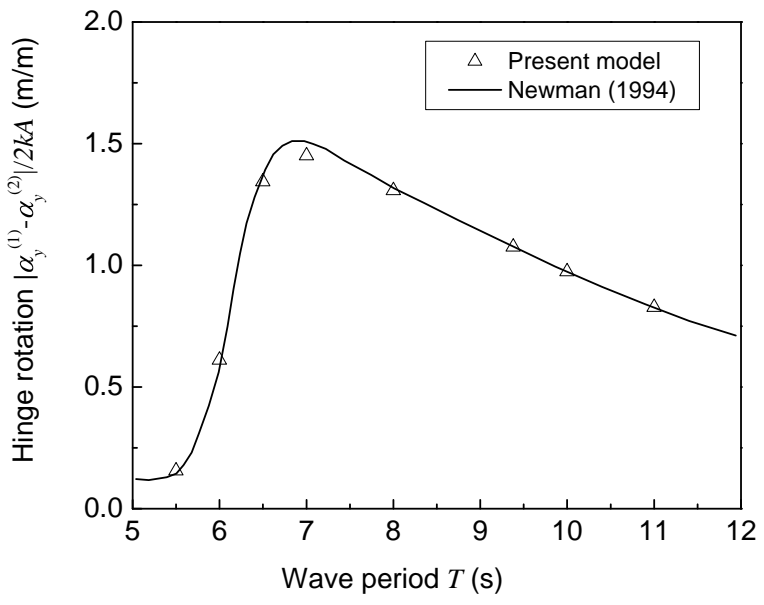

(b)

Figure 16: Comparisons of responses for two hinged barges: (a) vertical motion of hinge; (b) rotational angle of hinge in pitch

connection arm. The approach presented in Section 4 is applied to model the connections. Each of the two connection types is discussed in following sections.

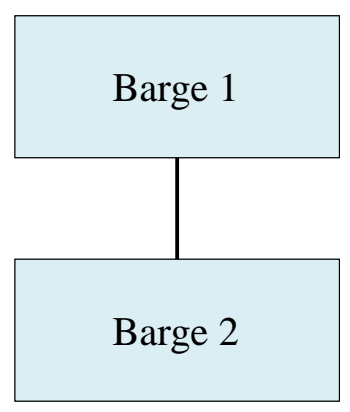

(a)

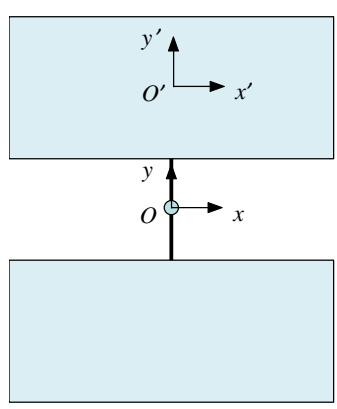

(b)

Figure 17: Plan view of side-by-side interconnected barges: (a) rigid connection and (b) middle-hinge connection

\subsection{Rigid connection}

The first type is two rigidly connected twin-barge system in Fig. 17(a) With a horizontal rigid bar connecting the two bodies, this system in another way can be treated as a single body of two hulls. A prototype of this system is a catamaran. Following the example of modeling rigid connections presented in Section 4 and submitting the particulars of the system configuration into Eq. 49, the constraint matrices 
for the two barges are

$$
\mathbf{K}_{1}=\left[\begin{array}{cccccc}
1 & 0 & 0 & 0 & 0 & 1 \\
0 & 1 & 0 & 0 & 0 & 0 \\
0 & 0 & 1 & -1 & 0 & 0 \\
0 & 0 & 0 & 1 & 0 & 0 \\
0 & 0 & 0 & 0 & 1 & 0 \\
0 & 0 & 0 & 0 & 0 & 1
\end{array}\right] ; \quad \mathbf{K}_{2}=\left[\begin{array}{cccccc}
1 & 0 & 0 & 0 & 0 & -1 \\
0 & 1 & 0 & 0 & 0 & 0 \\
0 & 0 & 1 & 1 & 0 & 0 \\
0 & 0 & 0 & 1 & 0 & 0 \\
0 & 0 & 0 & 0 & 1 & 0 \\
0 & 0 & 0 & 0 & 0 & 1
\end{array}\right]
$$

In order to investigate the influence of the connections, we also, for the purpose of comparison, simulate two side-by-side freely floating barges, with other configuration retained. We consider both a $0^{\circ}$ heading wave which propagates along the positive $x$ direction, i.e. head sea, and a $90^{\circ}$ heading wave propagating along the positive $y$ direction as beam sea.

\section{- Head sea}

Figure 18 plots the normalized (by the incident wave amplitude) free surface elevations in one wave period with the system subject to a $0^{\circ}$ heading wave at the frequency $\omega=6.0 \mathrm{rad} / \mathrm{s}$. Both the scattered and total wave fields are shown. As can be seen from the total wave field (right column of Fig. 18), no remarkably high surface elevation is observed on the free surface. Slightly amplified wave elevation appears only in the upwave surface of the barges, see $t=16.4 T$. Compared with the freely floating barges, the total wave field is not very much influenced by the rigid connection; this is possibly due to the fact that the barge motions are relatively small in this case. But definitely the barge motions are significantly changed by the rigid connection, compared to the case of two freely floating barges.

Figure 19 shows the motion responses of Barge 1 for both the cases of freely floating barges and rigidly connected barges in head sea at the frequency $\omega=6.0 \mathrm{rad} / \mathrm{s}$. As expected, motions of surge, heave and pitch are not much affected by the rigid connection due to symmetry. There in theory ought to be no relative surge, heave and pitch motions between Barges 1 and Barge 2, therefore the motions are not affected by the presence of rigid connection. This is because the constraint forces in the directions of surge, heave and pitch in the rigid connection are nearly zero. Meanwhile, the sway, roll and yaw motions become close to zero due to the rigid connection and the symmetric configuration of the system. This also is the case for Barge 2 . In addition, no drift effect should be expected for sway and yaw motions. Yet a small drift is observed in the yaw of Barge 1, as shown in Fig. 19(f). This may possibly be caused by the unstructured free surface mesh which makes the system not strictly symmetric about the $x$-axis. Comparisons of the motions between the two cases suggest that the constraint matrix model is essentially effective and can provide reasonable responses of the twin-barge system.

The constraint forces induced in the rigid bar are then of great interest. Figs. 20 and 21 plot the time histories of constraint forces and moments at the middle of the horizontal bar. The shear forces along the $x$ and $z$ directions are close to zero, due to the symmetry as explained in the motions. The constraint force $F_{y}$ 
(a)
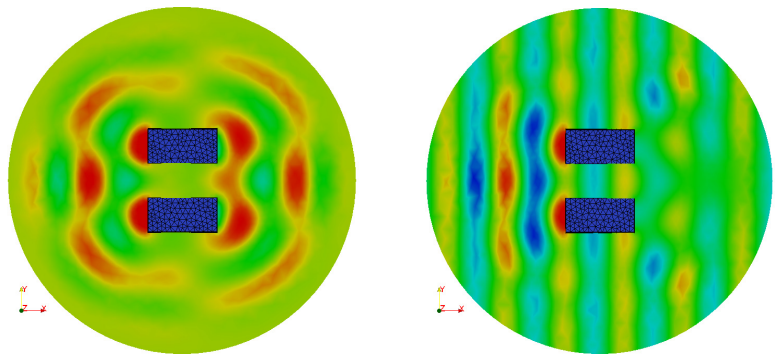

(b)
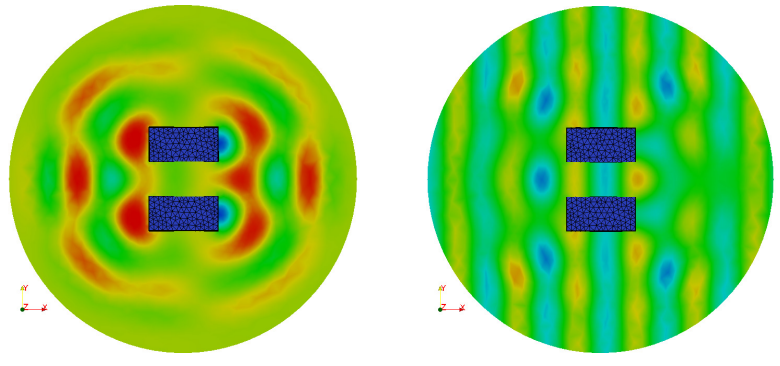

(c)
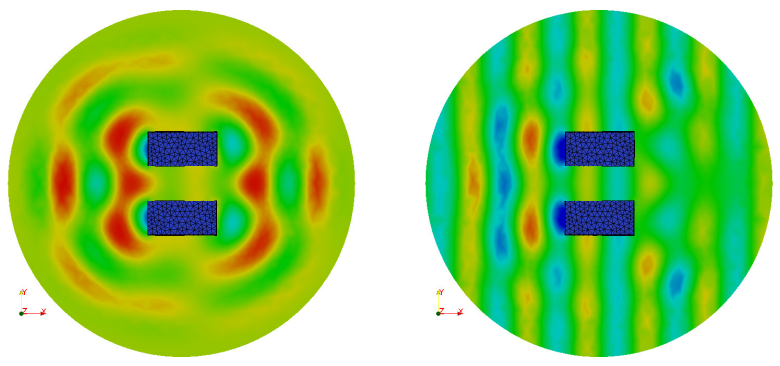

(d)
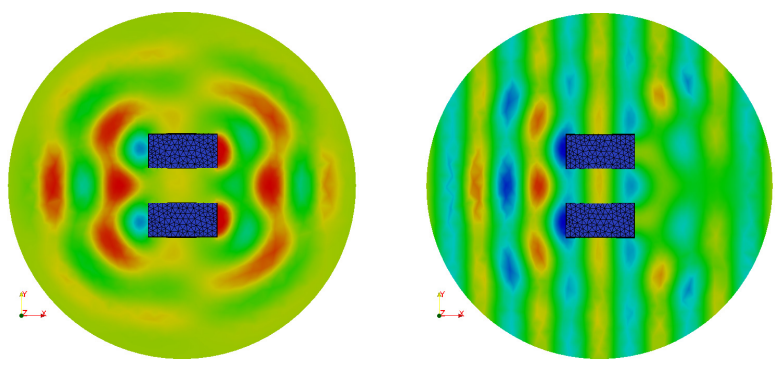

(e)
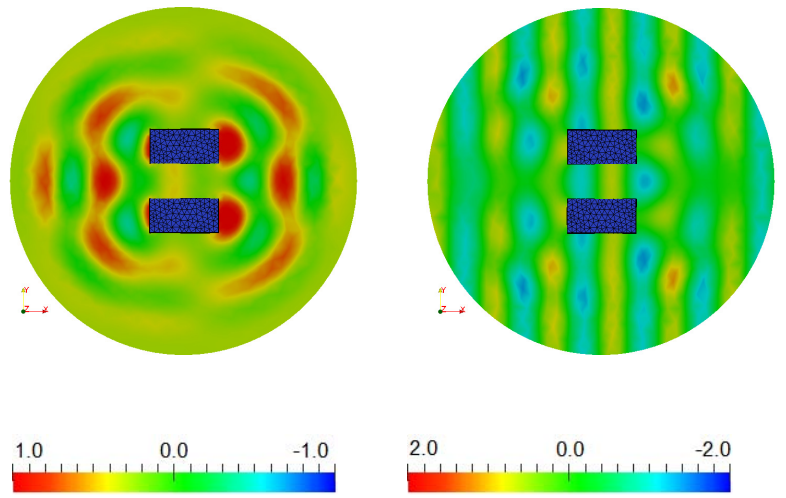

Figure 18: Free surface elevation in one period for side-by-side rigidly connected barges in head sea (rigid bar not shown), left column for scattered wave and right for total wave, at the time instants: (a) $t=16.4 T$; (b) $t=16.6 T$; (c) $t=16.8 T$; (d) $t=17.0 T$ and (e) $t=17.2 T$ 


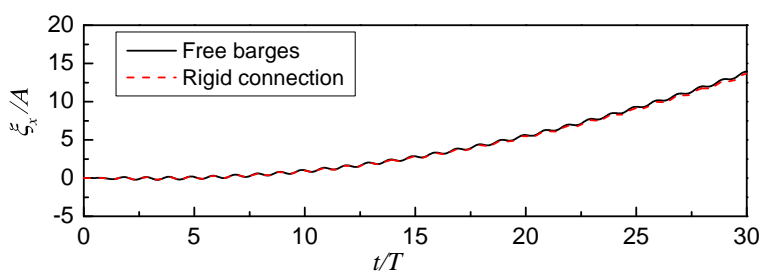

(a) Surge

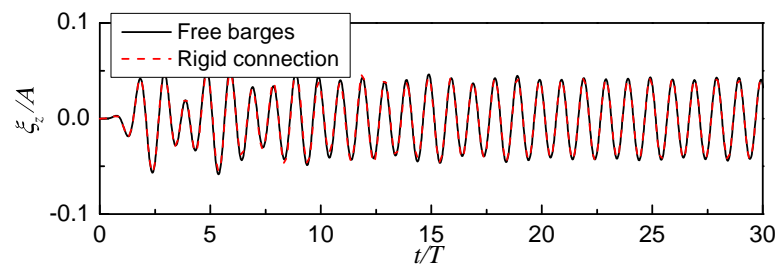

(c) Heave

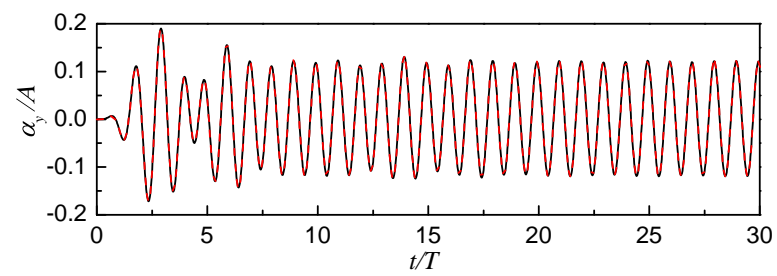

(e) Pitch

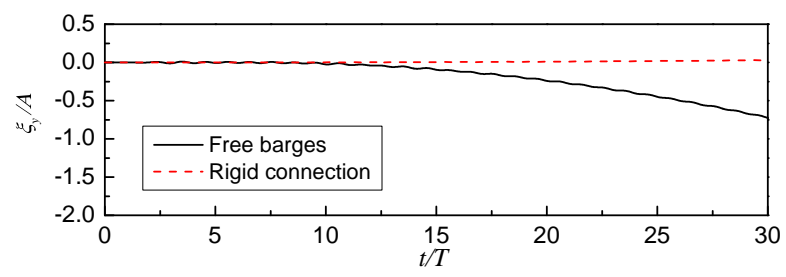

(b) Sway

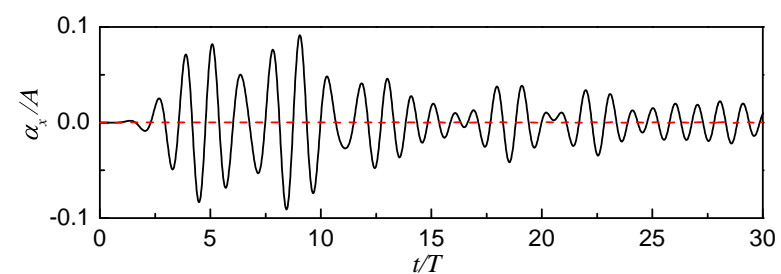

(d) Roll

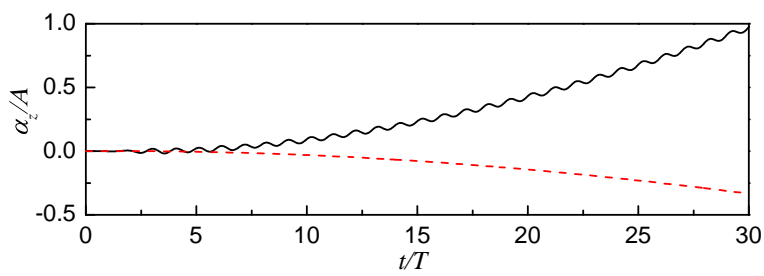

(f) Yaw

Figure 19: Comparisons of motions of Barge 1 for two side-by-side free barges and rigid connected barges subject to a $0^{\circ}$ incident wave of frequency $\omega=6.0 \mathrm{rad} / \mathrm{s}$ : (a) Surge; (b) Sway; (c) Heave; (d) Roll; (e) Pitch and (f) Yaw

along the bar is almost equal to the hydrodynamic sway force acting on the barges. In addition, the mean $F_{y}$ is nonzero and its direction, pushing the side-by-side barges toward or outward each other, depends on the incident wave frequency. For constraint moments in Fig. 21, the yaw moment $M_{z}$ is much higher than the roll moment $M_{x}$ as well as pitch moment $M_{y}$ which are near zero. It is not surprising to see the near zero pitch moment since there is no relative motion in pitch between the two barges. The yaw motions of Barges 1 and 2 tend to be opposite (this can be observed from the case of free barges), which generates a large yaw moment $M_{z}$ at the rigid connection to resist their antisymmetric movement. However, their tendency of antisymmetric roll motion does not result in a very large moment $M_{x}$ at the connection. In the perspective of the connection strength, the bending moment about the $z$-axis is more crucial than that about the $x$-axis. This would be an important practical concern if two side-by-side barges were rigidly connected.

\section{- Beam sea}

We next consider these two rigidly connected barges subject to a $90^{\circ}$ heading wave of frequency $\omega=6.0 \mathrm{rad} / \mathrm{s}$. The scattered wave field in one wave period at steady state is displayed in Fig. 22 . Compared with the head sea case, wave surface between the two barges are more disturbed. Careful scrutiny of the scattered elevations in-between shows that a near standing wave occurs. Unlike the longitudinal wave resonance in a narrow gap 


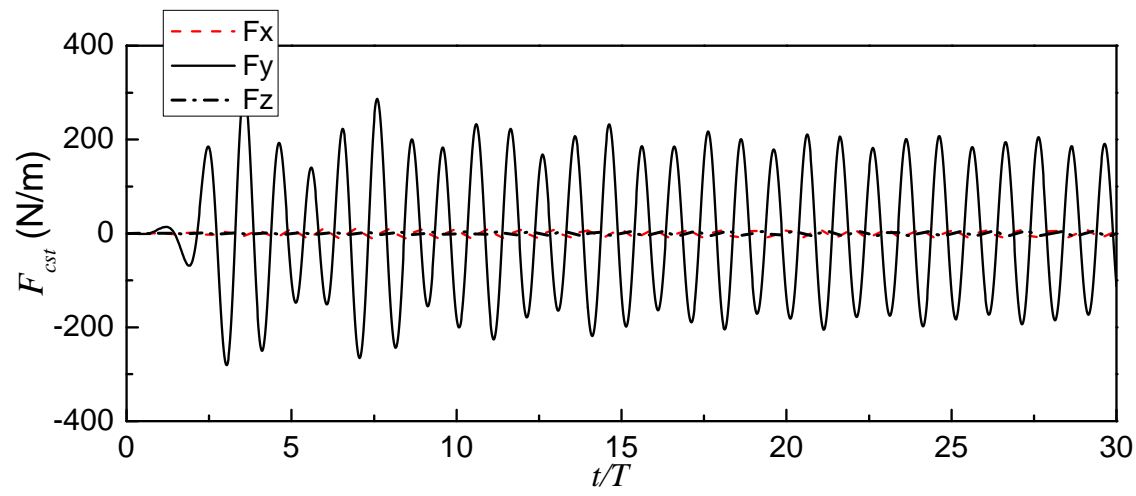

Figure 20: Constraint forces at the middle of the rigid connection bar for side-by-side rigidly connected barges in head sea

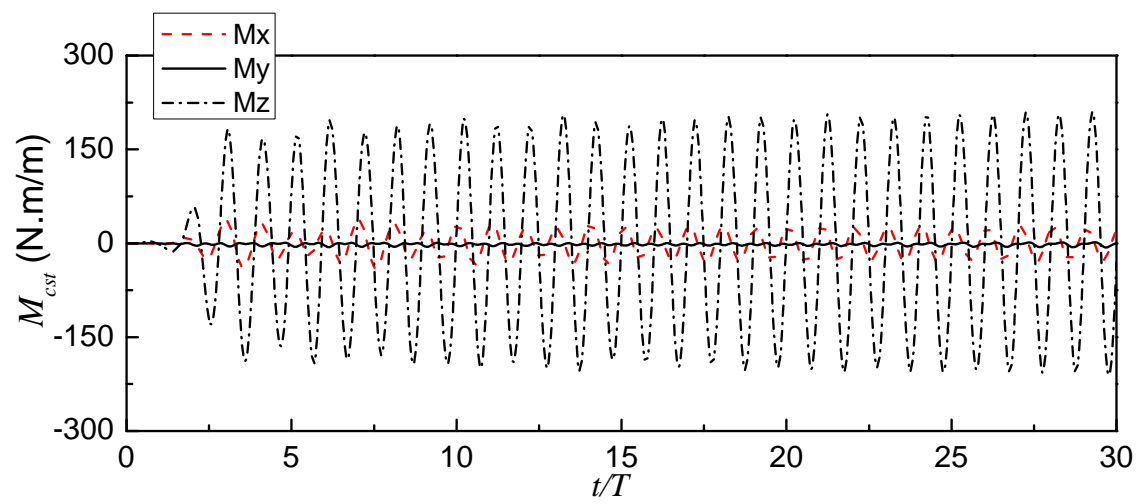

Figure 21: Constraint moments at the middle of the rigid connection bar for side-by-side rigidly connected barges in head sea

which has been extensively investigated in Feng and Bai (2015), this can be explained as a fluid sloshing between two vertical walls. Apparently it depends much on the wave length/frequency and gap distance. As we focus on the influence of the interconnection on the barge motions, the sloshing issue is out of the scope of this study hence is not discussed further here.

The presence of the rigid connection is expected to have more influence on the barge motions in the case of beam sea. The surge, pitch and yaw motions of both Barge 1 and Barge 2 are quite close to zero in a wave of $90^{\circ}$ incidence. We discuss only motions in the other three DOFs. Figs. 23 and 24 show the responses of sway, heave and roll of the upwave Barge 2 and leeside Barge 1, respectively. The harmonic components in the sway response in Fig. 23(a) are almost equal to zero. Remarkably the sway drift drops nearly half of that of the free barges. This is reasonable because the rigidly connected system has a lower mean acceleration than the upwave freely floating barge. On the contrary, this is not the case for the leeside Barge 1, plotted in Fig. 24(a), With two free barges, the leeside Barge 1 is well sheltered by Barge 2 such that Barge 1 has nearly no sway motion and drift. Whereas in the rigidly connected system the upwave and leeside barges 


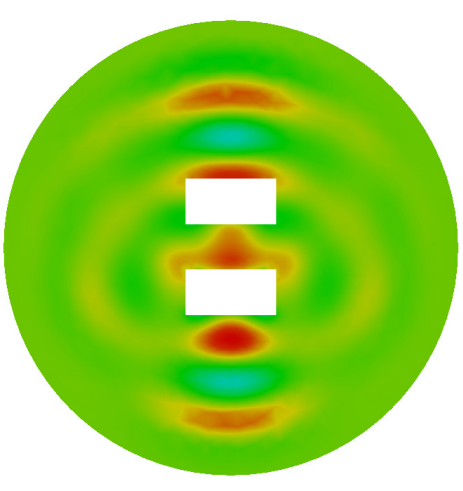

(a)

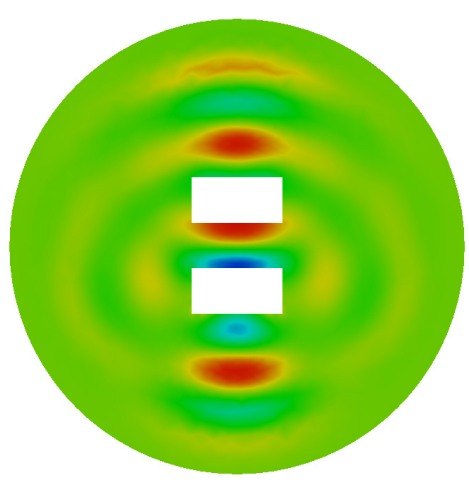

(c)

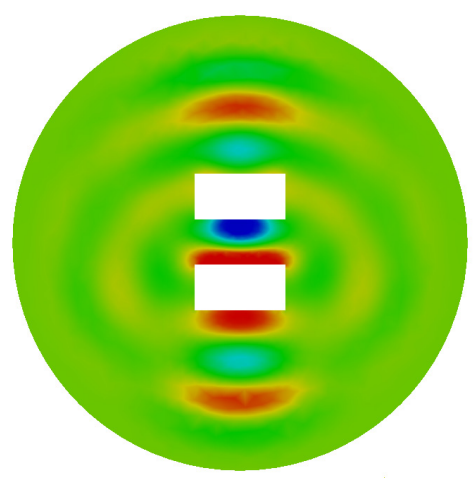

(e)

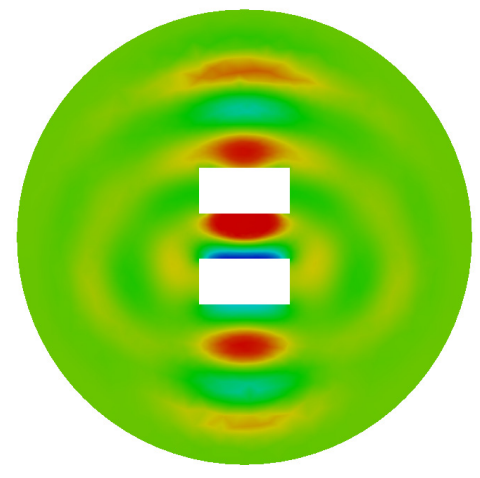

(b)

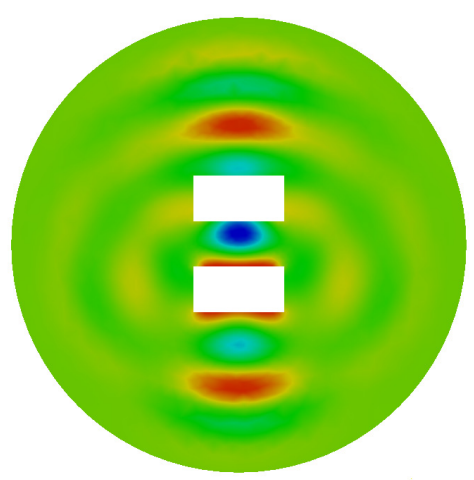

(d)

Figure 22: Scattered wave elevation in one period for side-by-side rigidly connected barges (rigid bar not shown) in beam sea at the time instants: (a) $t=16.4 T$; (b) $t=16.6 T$; (c) $t=16.8 T$; (d) $t=17.0 T$ and (e) $t=17.2 T$

share a same sway motion.

For heave motions, it is surprising that both the heaves of Barge 2 in Fig. 23(b) and Barge 1 in Fig. 24(b) are slightly increased by the presence of the rigid connection. This could be attributed to the roll motion of the system, rotating as a single body about the middle of the horizontal bar, whereas the roll center of each 


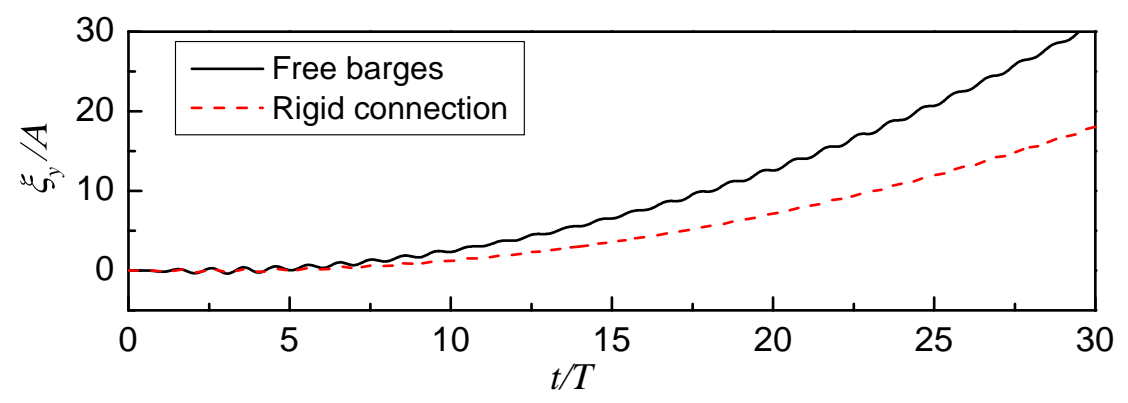

(a)

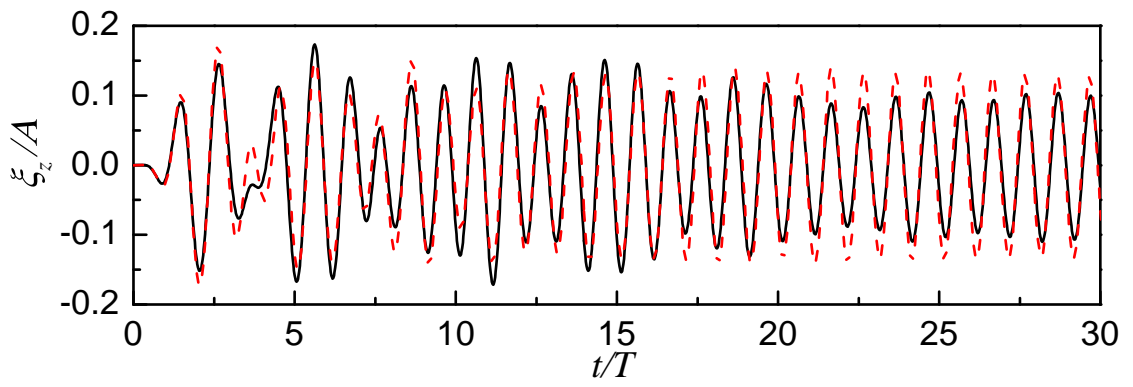

(b)

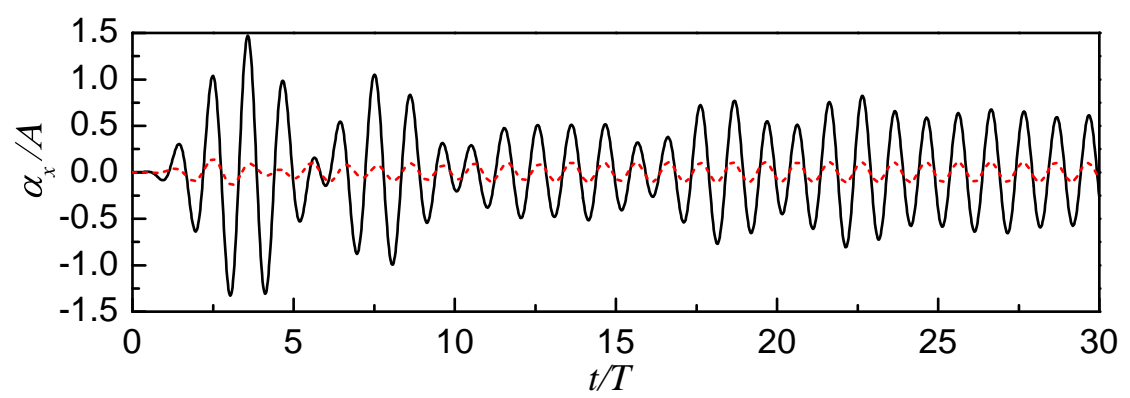

(c)

Figure 23: Motions of upwave Barge 2 for two rigidly connected barges in beam sea, with comparisons to two free barges: (a) Sway; (b) Heave and (c) Roll

free barge is at its center of gravity. The system roll results in additional vertical displacement at each end of the horizontal bar, which is accounted for the increased vertical motion of each barge. The roll motions of the two rigidly connected barges are identical, yet much moderated, comparing with the free barges. In Fig. 23(c) a significant drop of roll motion of the upwave Barge 2 occurs due to the rigid connection. For the leeside Barge 1 in Fig. 24(c), although the steady-state roll motion is comparable with the free barges, the transient response before around $20 \mathrm{~T}$ for the case of free barges could be several times higher than the case of rigid connection. In beam sea, a similar conclusion as in the situation of head sea can be drawn in the perspective of roll motion. That is, for two side-by-side barges in both head sea and beam sea, the rigid interconnection helps to significantly diminish the roll motions of freely floating barges. It is an important 
benefit since vessel roll motion in practice is more critical than motions in other DOFs. Nevertheless, it ought to be borne in mind that the roll motion predicted via the potential flow model may not be realistic because the viscous roll damping is not considered in this paper.

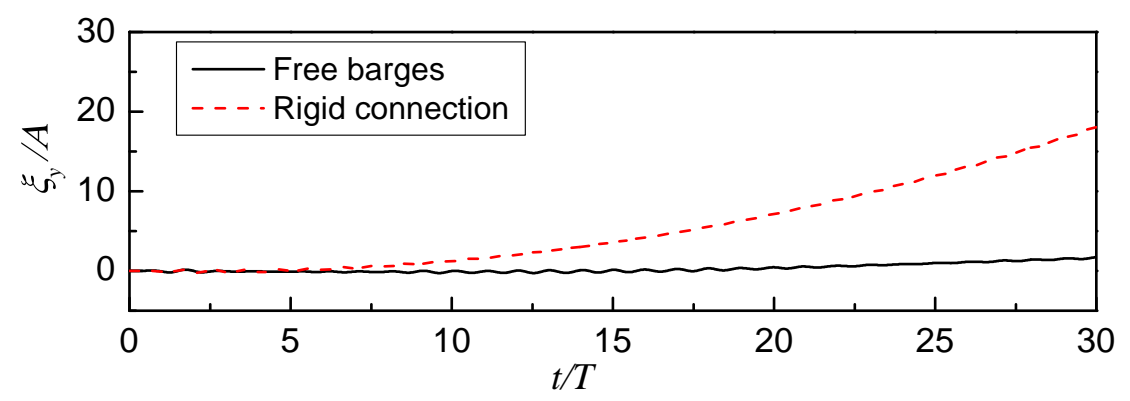

(a)

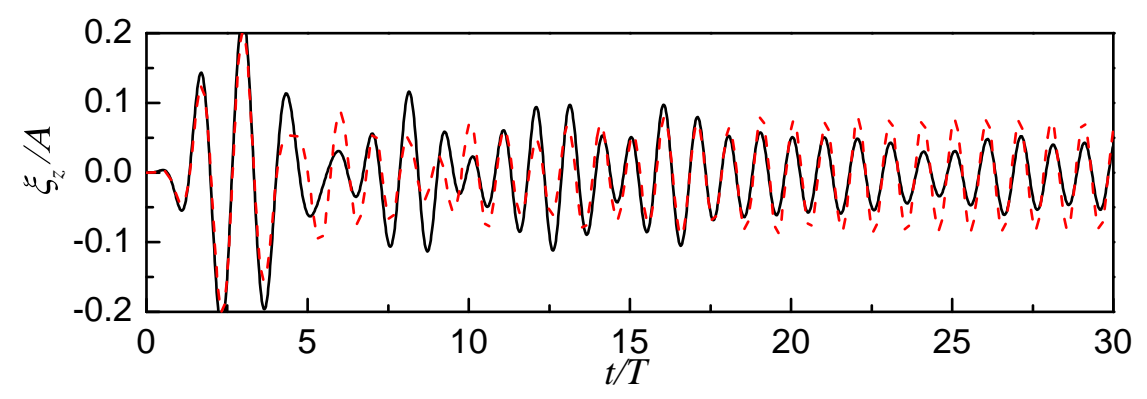

(b)

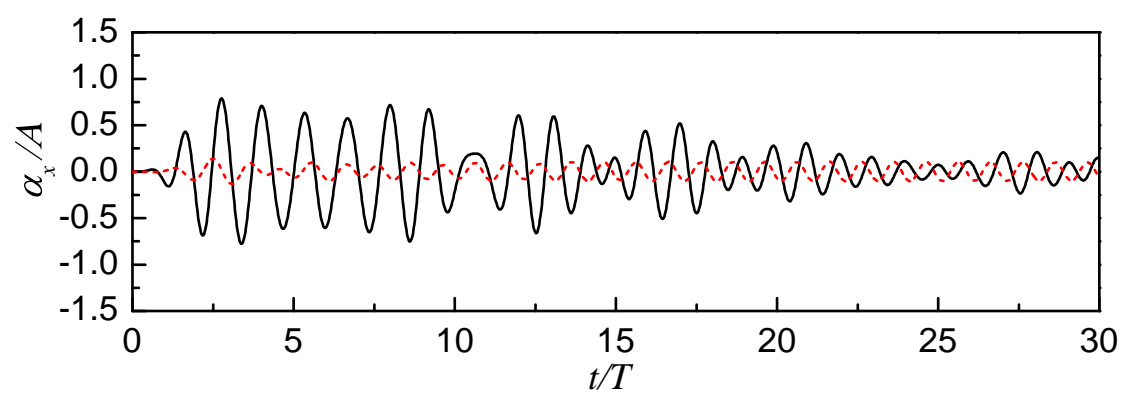

(c)

Figure 24: Motions of leeside Barge 1 for two rigidly connected barges in beam sea, with comparisons of two free barges: (a) Sway; (b) Heave and (c) Roll

Besides, we notice that the roll motions for the upwave and leeside barges have a phase difference in the case of free barges. While the roll responses of the two free barges are in anti-symmetrical direction in head sea, their phase difference in beam sea depends on the ratio of wave length to their separation distance. With the rigid connection, the two barges naturally have a same roll motion, both in amplitude and phase. A phase difference, especially the out-of-phase, generates a higher relative motion which endangers the side-by-side operations. The rigid connection to somewhat stabilizes the system in terms of relative motions between 
two bodies.

Despite the effect of mitigating relative motions, very high constraint forces are generated in the rigid bar in the beam sea condition. Figs. 25 and 26 show respectively the forces and moments at the middle of the rigid bar. The in-line force, $F_{y}$, now is over ten times that in the case of head sea plotted in Fig. 20. This is due to the fact that the sway wave force in beam sea is much higher than that in head sea. A shear force $F_{z}$ is also excited, though not as high as the in-line force. Unlike in head sea in Fig. 21, where a large bending moment $M_{z}$ is induced to resist the relative yaw motion of the barges, a nearly twice the bending moment about the $x$-axis is generated in beam sea. Again one must pay much attention to his bending moment $M_{x}$ if adopting a rigid connection to stabilize the two barges.

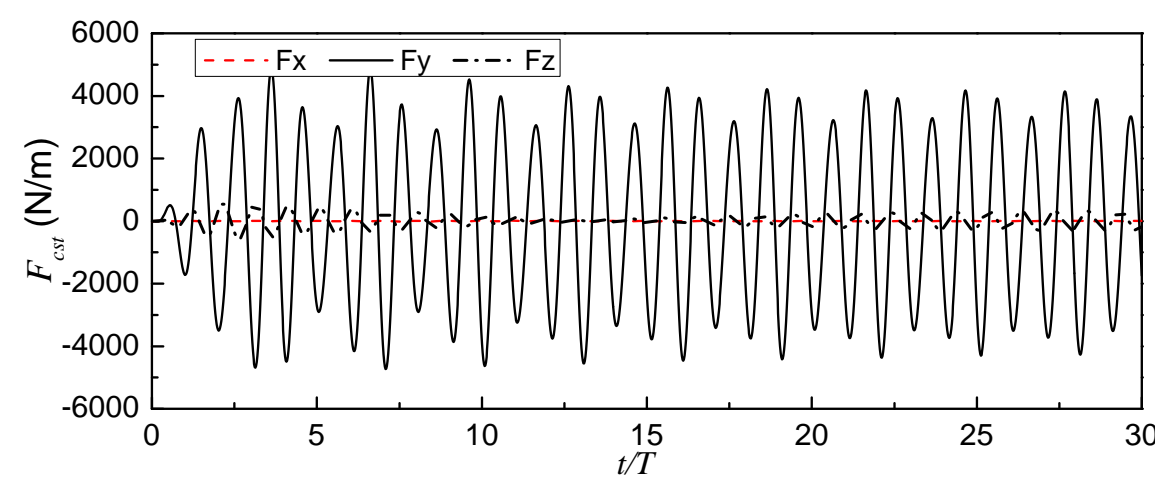

Figure 25: Constraint forces at the middle of the rigid connection bar for side-by-side rigidly connected barges in beam sea

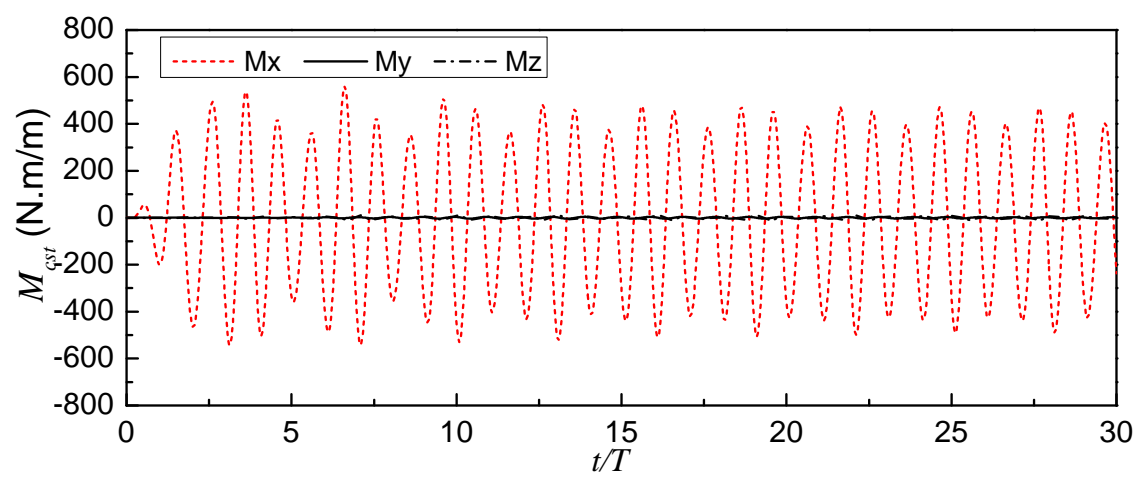

Figure 26: Constraint moments at the middle of the rigid connection bar for side-by-side rigidly connected barges in beam sea

\subsection{Middle-hinge connection}

The second type is a middle-hinged twin-barge system, where the barges are connected by a hinge at the middle of a horizontal bar, as shown in Fig. 17(b). In the practical point of view, this connection is 
more convenient and efficient than the rigid connection. The side-by-side barges can be easily connected and disconnected via the joint hinge according to the requirement. Similar to the case of rigid connection, we need to construct the constraint matrices for the two barges. As the rotational constraints are freed at the hinge, the corresponding coefficients in the constraint matrix become zero. The resultant constraint matrices are written as

$$
\mathbf{K}_{\mathbf{1}}=\left[\begin{array}{cccccc}
1 & 0 & 0 & 0 & 0 & 1 \\
0 & 1 & 0 & 0 & 0 & 0 \\
0 & 0 & 1 & -1 & 0 & 0 \\
0 & 0 & 0 & 0 & 0 & 0 \\
0 & 0 & 0 & 0 & 0 & 0 \\
0 & 0 & 0 & 0 & 0 & 0
\end{array}\right] ; \quad \mathbf{K}_{2}=\left[\begin{array}{cccccc}
1 & 0 & 0 & 0 & 0 & -1 \\
0 & 1 & 0 & 0 & 0 & 0 \\
0 & 0 & 1 & 1 & 0 & 0 \\
0 & 0 & 0 & 0 & 0 & 0 \\
0 & 0 & 0 & 0 & 0 & 0 \\
0 & 0 & 0 & 0 & 0 & 0
\end{array}\right]
$$

Note that the 'zero' rows in the constraint matrices must be eliminated while forming the coupled motion equations, saying Eq. (48). As the rotational moments are freed at the hinge, only three constraint forces need to be solved. After eliminating the 'zero' rows in the constraint matrices, we are to solve a 15 equation system for this coupled motions.

\section{- Head sea}

We impose the same incident wave into the tank. The condition of head sea is firstly considered. Compared with the rigid connection, the middle-hinge connected barges are not restricted in their roll and yaw movements. Particularly for the symmetric case in head sea the pitch motions of two barges are the same. It is found that the roll motions for the middle-hinge connected barges are almost in coincidence with the two freely floating barges (see Fig. 27(a)], this is due to the fact that the free barges are rolling anti-symmetrically and no bending moment is generated in the middle-hinge connection, under the small rotational motion assumption. The yaw motion of Barge 1 is plotted in Fig. 27(b). Harmonic analysis shows that the first order motion is almost same as in the case of free barges. However, the drift of yaw is much reduced compared with the free barges. We have discussed in the simulation of rigidly connected barges that a remarkable bending moment in the horizontal bar is induced as a balance of the hydrodynamic pressure moment on the barges. This is not an issue here for the middle-hinge connection and can be considered as one main advantage over the rigid connection.

The constraint forces at the hinge are presented in Fig. 28, similar to the force responses for the rigid connection in Fig. 20. There is no constraint moment in the middle-hinge connection.

\section{- Beam sea}

We now present the case of the two middle-hinged barges in beam sea. In the concern of interconnection, the middle-hinge connection has no constraint in relative rotational motions of the barges while the rigid connection engages the two barges as a single body. In such circumstance, the surge, sway and heave motions of middle-hinged barges in beam sea are quite similar to those of rigidly connected barges. As there is almost 


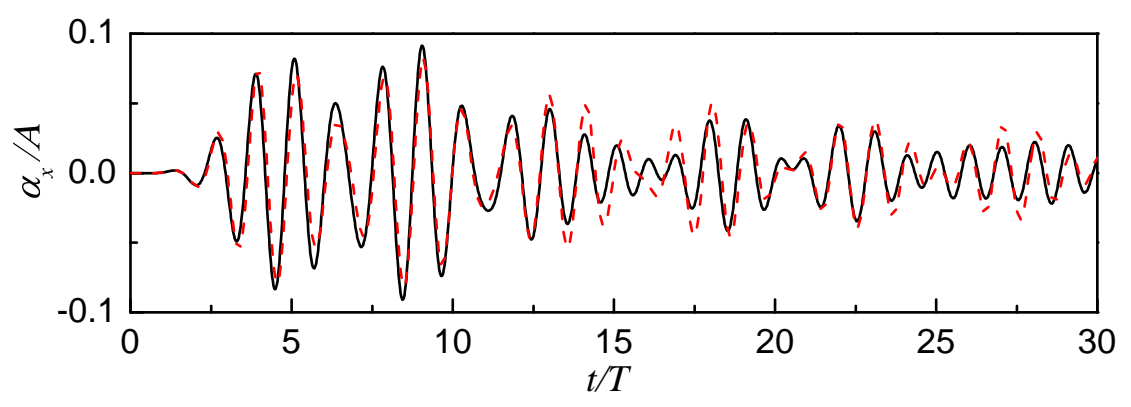

(a) Roll

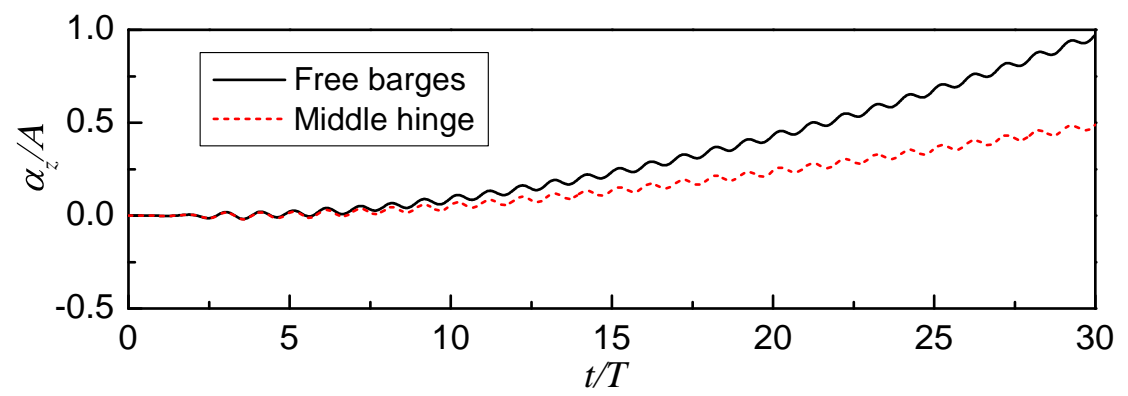

(b) Yaw

Figure 27: Comparisons of motions of Barge 1 for two side-by-side free barges and middle-hinge connected barges subject to a $0^{\circ}$ incident wave of frequency $\omega=6.0 \mathrm{rad} / \mathrm{s}$

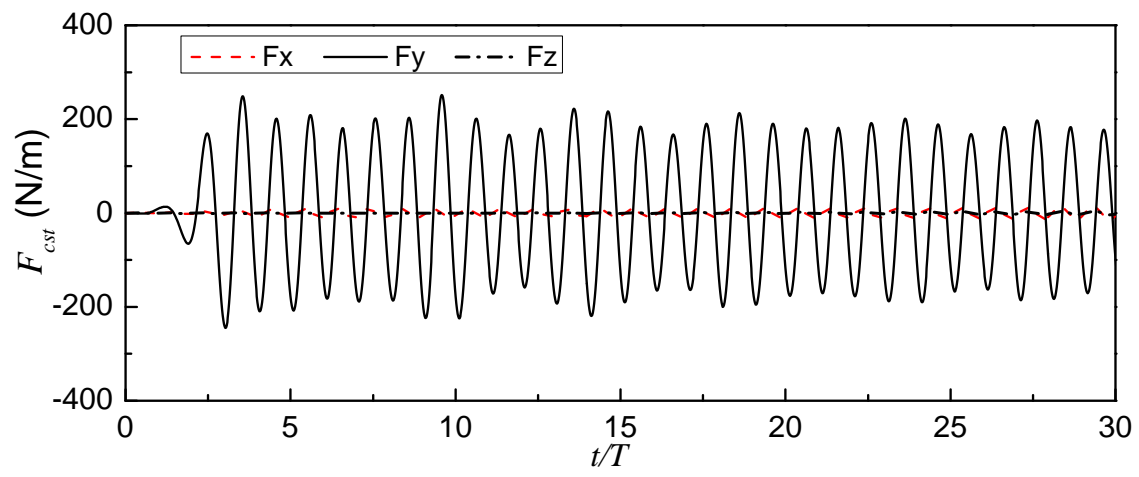

Figure 28: Constraint forces at the hinge for side-by-side middle-hinge connected barges in head sea

no pitch and yaw for the barges in beam sea, the effect on roll motion is the major difference between the rigid and middle-hinge connections. Figs. 29(a) and 29(b) show the roll responses of the leeside Barge 1 and upwave Barge 2 respectively, with comparisons against the case of two free barges. Note that the steady-state responses are achieved after around 20 wave periods. The roll amplitude of the upwave Barge 2, at nearly the steady state, slightly reduces due to the middle-hinge connection. Nevertheless, this reduction effect of the middle-hinge connection is not as significant as that of the rigid connection shown in Fig. 23(c), On the contrary, the middle-hinge connection amplifies in a small scale the roll response of the leeside Barge 1. 
This is reasonable if we examine carefully the phase of the roll motion. We observe that the phases of roll motions are the same for the upwave Barge 2 with/without the connection while there apparently exists a phase difference for the leeside Barge 1. As we have discussed previously, there naturally is a phase difference for roll motion between the upwave and leeside barges. Due to the middle-hinge connection, Barge 1 and Barge 2 are now rolling exactly out-of-phase. This has somewhat mitigated the discrepancy of roll motions between the two barges.

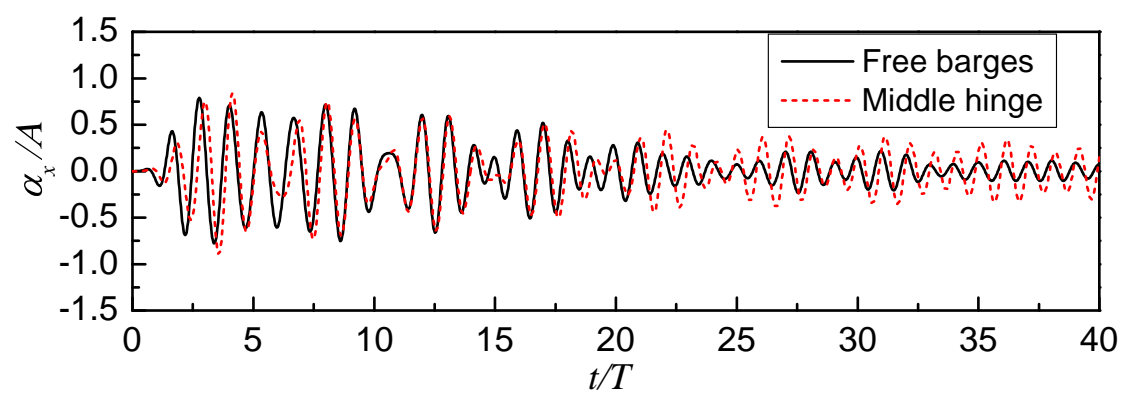

(a)

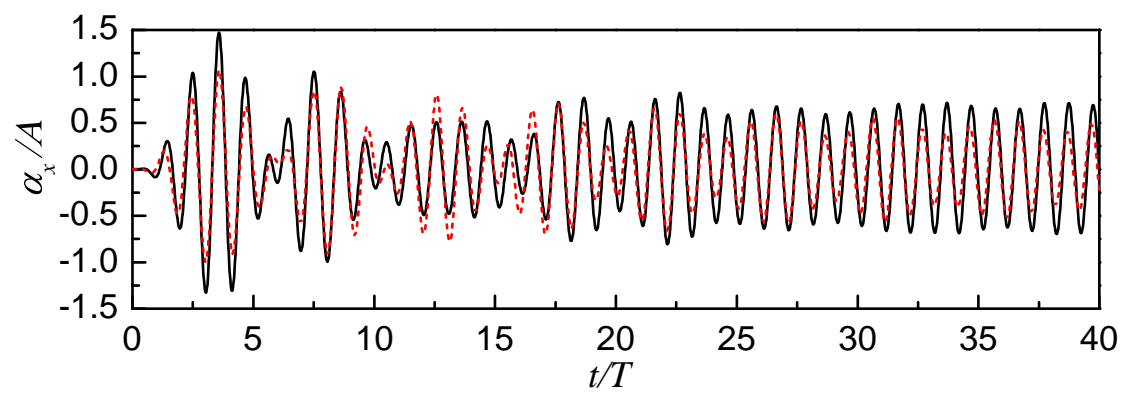

(b)

Figure 29: Roll motions of two side-by-side middle-hinge connected barges in beam sea: (a) Barge 1 and (b) Barge 2

In this complicated problem of wave interactions with multiple floating bodies, the motion variation induced by the interconnection in return could change the hydrodynamic forces on the barges. Particularly the heave force and roll moment on the barges in beam sea are expected to be much influenced because the motions in these directions are considerably subject to the interconnection constraints. Fig. 30 shows the hydrodynamic force and moment on the upwave Barge 2 in the heave and roll directions. It is found that the variation (increase or decrease) of the hydrodynamic forces induced by the middle-hinge connection follows the trend in the corresponding motions, i.e. the heave force on Barge 2 increases while the roll moment decreases. It is also true for the forces on the leeside Barge 1, shown in Fig. 31. Both the heave force and roll moment on Barge 1 (steady state) have been enlarged over $50 \%$ of the forces on the free barges, which indeed is the case for their corresponding motions.

The constraint forces at the hinge are close to those at the middle point of the horizontal bar in the case of rigid connection, except that there is no resistant moment in the middle-hinge connection. The responses 


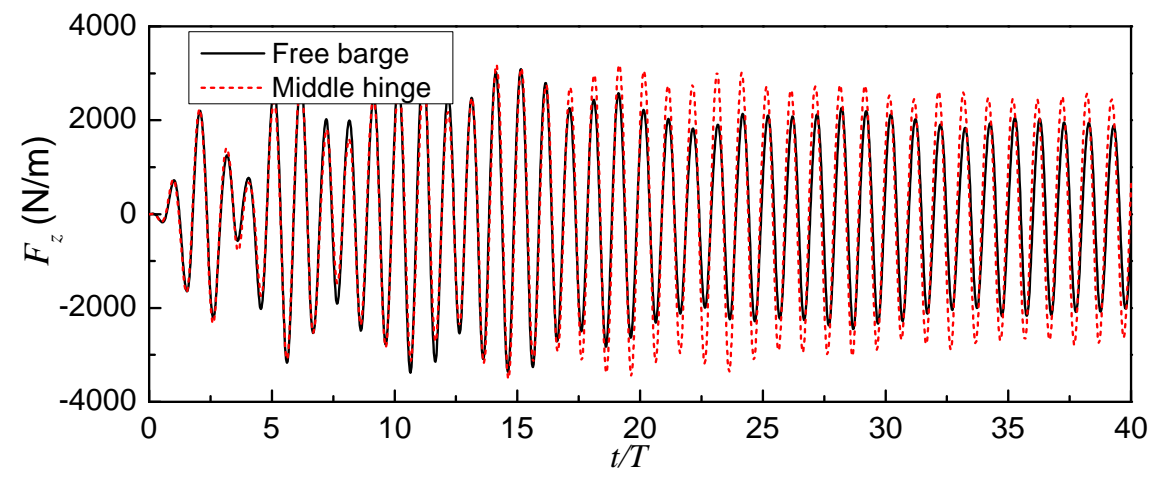

(a)

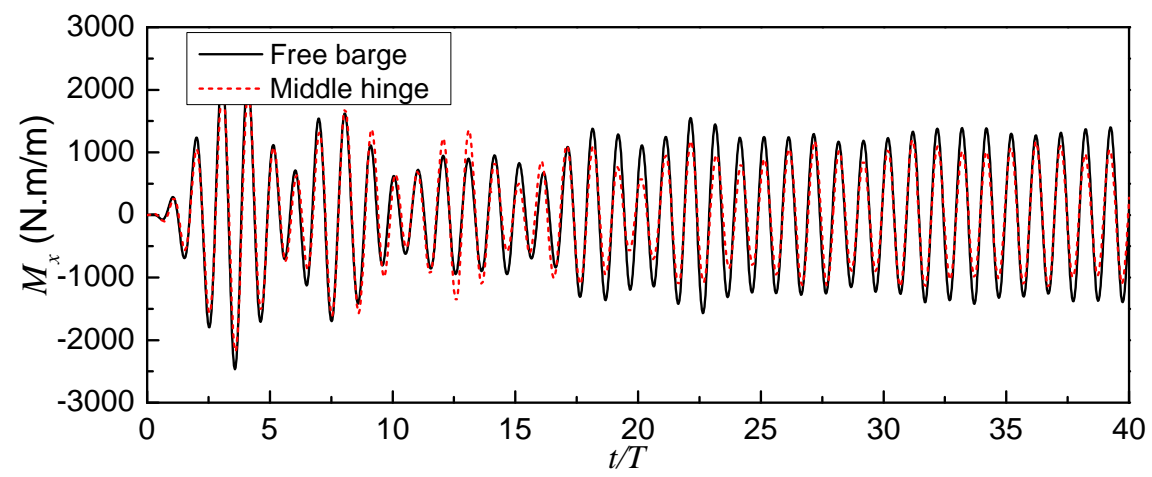

(b)

Figure 30: Hydrodynamic forces on upwave Barge 2 for two side-by-side middle-hinge connected barges in beam sea: (a) heave force $F_{z}$ and (b) roll moment $M_{x}$

of the forces are presented in Fig. 32. The in-line force $F_{y}$ dominates and a small shear force $F_{z}$ is also present.

\subsection{Nonlinear effect}

Apart from the capability of time-domain simulation of the interconnected floating system, a key nature of the present numerical model is its ability to capture the nonlinear effect caused by the nonlinear free water surface. As a matter of fact, the nonlinear effect cannot be evaluated using linear simulations, which, however, are commonly used in the marine offshore industry. To demonstrate the advanced feature of capturing nonlinearity, we now consider again the case of the middle-hinge connected barges. The nonlinearity is investigated by increasing the incident wave steepness $k A$, where $k$ is wave length and $A$ is wave amplitude. Fig. 33 compares the time history of the scattered wave elevation in simulations using mild wave $k A=0.005$ and relatively steeper wave $k A=0.1$ at the frequency $\omega=6.0 \mathrm{rad} / \mathrm{s}$ in head sea. Responses at two locations are plotted, i.e. center of the system $(0.0,0.0)$ in Fig. 33(a) and its upstream position $(-0.3,0.0)$ in Fig. 33(b), A near-sinusoidal response (dash line) is observed for $k A=0.005$ in Fig. 33(a), whereas for 


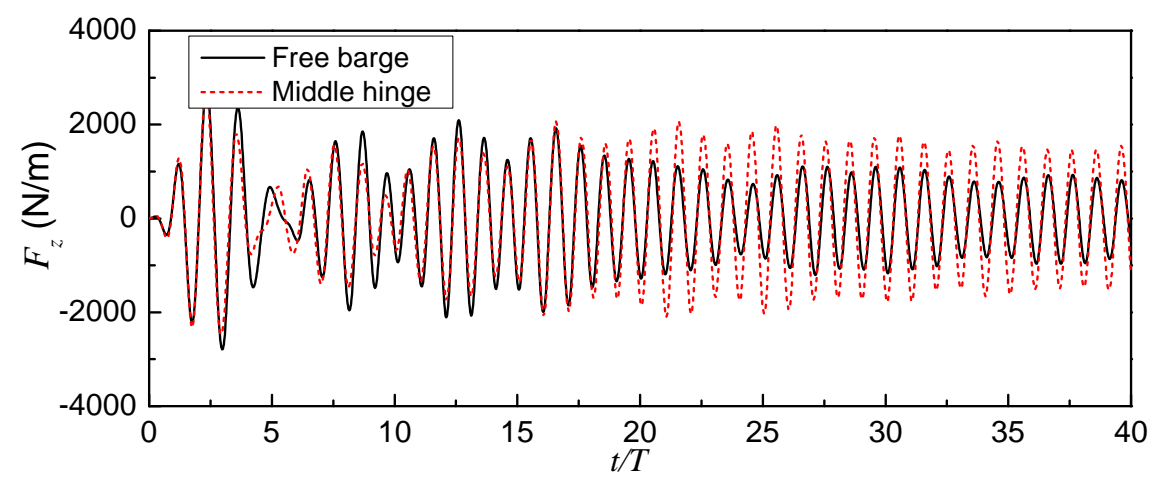

(a)

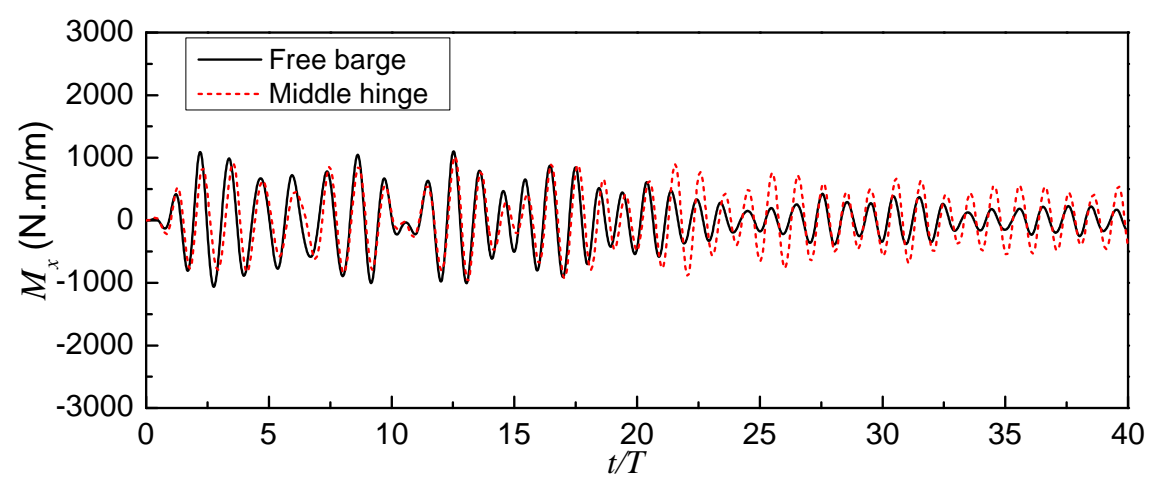

(b)

Figure 31: Hydrodynamic forces on leeside Barge 1 for two side-by-side middle-hinge connected barges in beam sea: (a) heave force $F_{z}$ and (b) roll moment $M_{x}$

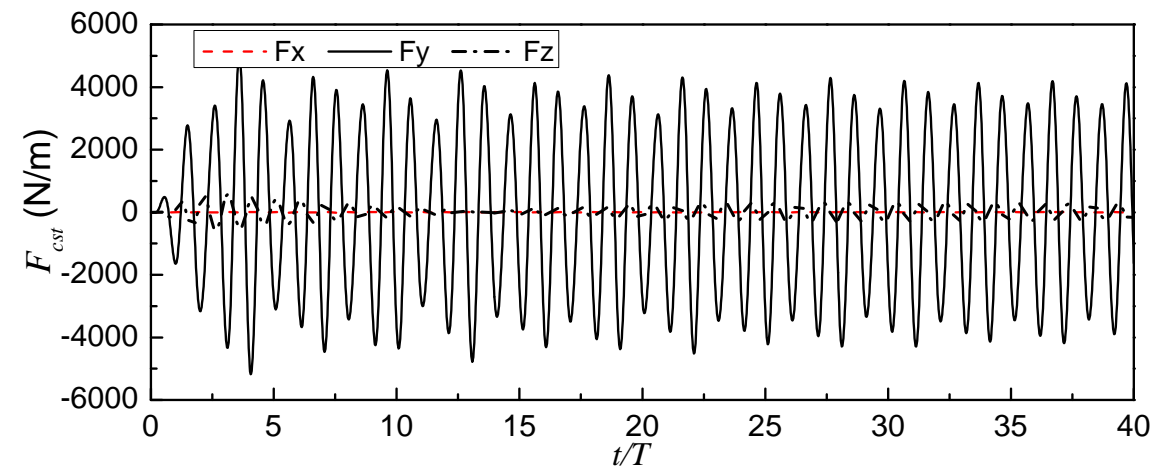

Figure 32: Constraint forces at the hinge for side-by-side middle-hinge connected barges in beam sea 
$k A=0.1$ a slightly sharpened wave crest and a flattened trough are shown. The nonlinear effect is also observed in Fig. 33(b), where the response for $k A=0.1$ presents a flattened crest and a sharpened trough. These are because higher-order components become more significant in the case of steeper wave $k A=0.1$. The comparison between the two wave steepnesses clearly highlights the effect of nonlinearity. This is further demonstrated in the responses of wave forces and body motions below.

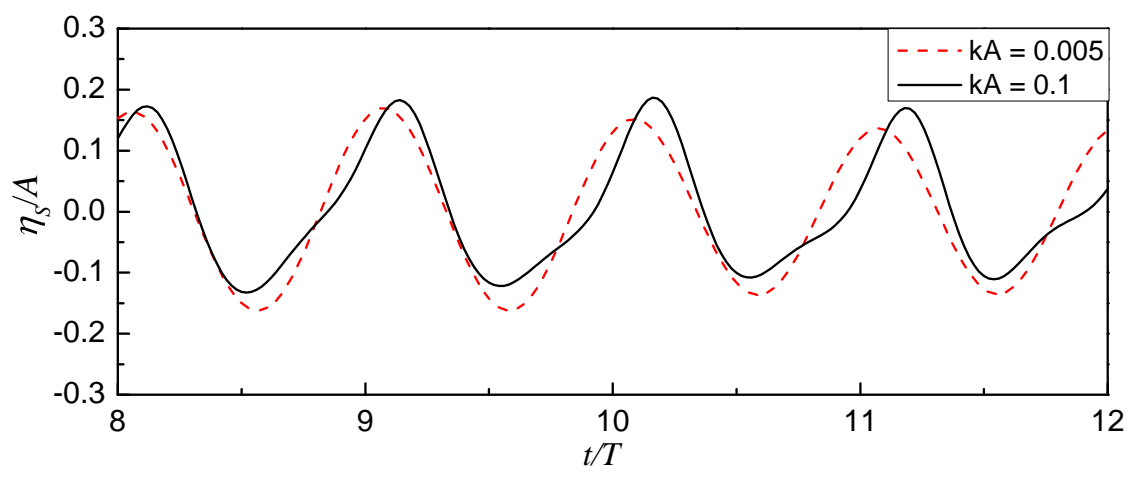

(a)

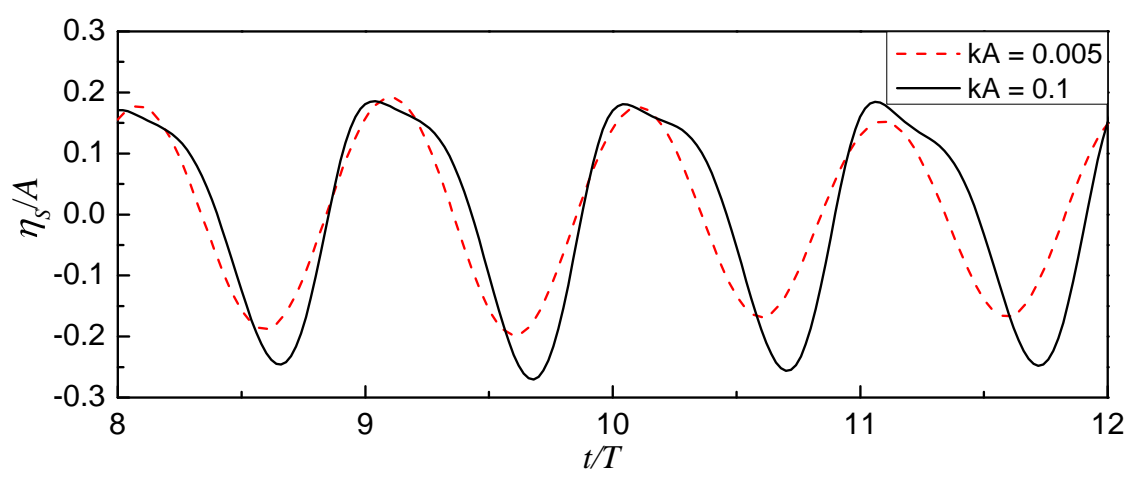

(b)

Figure 33: Scattered wave elevations in the case of two side-by-side middle-hinge connected barges in head sea, with incident waves of different steepnesses at frequency $\omega=6.0 \mathrm{rad} / \mathrm{s}$, at locations: (a) $x=0.0 \mathrm{~m}, y=0.0 \mathrm{~m}$ and (b) $x=-0.3 \mathrm{~m}, y=0.0 \mathrm{~m}$

In order to evaluate the effect of increased wave steepness on the system hydrodynamics, the time histories of surge, heave, pitch and yaw of Barges 1 are presented in Fig. 34. The simulation using an intermediate wave $k A=0.05$ is also included. With the increase of wave amplitudes/steepnesses, the barge accelerations in surge and yaw are dramatically enlarged, as shown in Figs. 34(a) and 34(d), directly due to the enlarged second-order drift forces in the respective directions. For the motions of heave and pitch in Figs. 34(b) and $34(\mathrm{c})$, in which the drift forces are zeros, the nonlinear effect on the response maxima seems limited. What is noteworthy is the phase shift between the mild and steep waves. Only a negligible phase difference is presented at the time instant $t=8 T$ while the phase difference becomes distinct at $t=12 T$. This is 
expected as a result of the dramatically enlarged surge motion in the steeper wave $k A=0.1$, i.e. the barges are drifting to downstream faster than those in the mild wave $k A=0.005$.

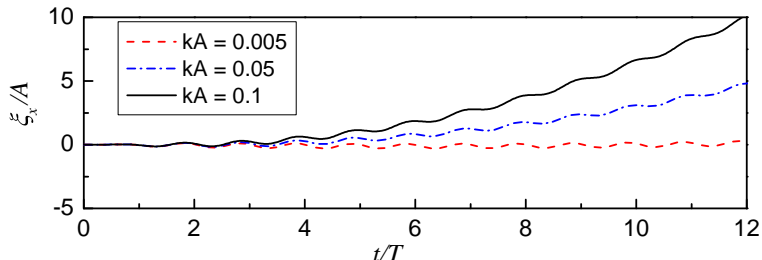

(a) Surge

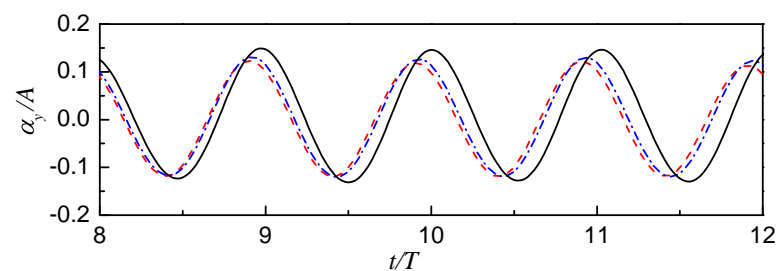

(c) Pitch

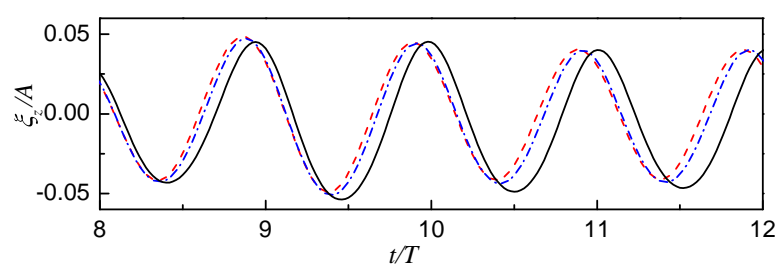

(b) Heave

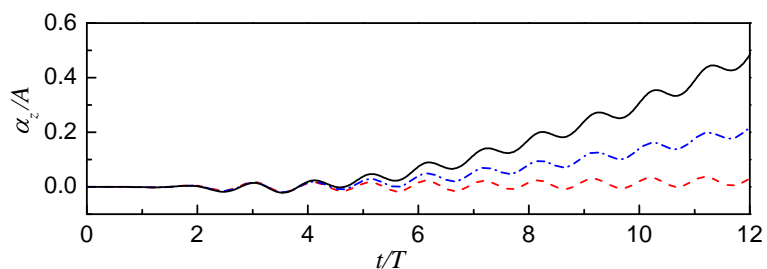

(d) Yaw

Figure 34: Motion responses of Barge 1 in the case of two middle-hinge connected barges in head sea, with incident waves of different steepnesses at frequency $\omega=6.0 \mathrm{rad} / \mathrm{s}$ : (a) Surge; (b) Heave; (c) Pitch and (d) Yaw

We then perform harmonic analysis to give a clear view of the nonlinearities associated with the free surface conditions as well as the body boundary conditions. Fig. 35(a) shows the comparison of vertical heave force on Barges 1 in the waves of increased steepness. It is again found that there are significant components at frequencies other than the dominant incident wave frequency for $k A=0.1$. Performing the FFTs on the time series in Fig. 35(a) results in the force amplitude spectra in Fig. 35(b) for $k A=0.005$ and Fig. 35(c) for $k A=0.1$. Note that the frequency in the spectrum plot is normalized by the incident wave frequency $\omega_{0}=6.0 \mathrm{rad} / \mathrm{s}$. Clearly for $k A=0.005$ the components at frequencies other than $\omega / \omega_{0}=1$ are almost negligible, whereas for $k A=0.1$ there appears a significant component at $\omega / \omega_{0}=2$, corresponding to a double frequency. This double-frequency component (second harmonic) is about $1 / 3$ the dominantfrequency component (first harmonic), and could not be ignored. Third and even higher harmonics play a minor role in shaping the nonlinear response in Fig. $35(\mathrm{a})$ for $k A=0.1$.

Simulations then are carried out over a range of wave frequencies $5-7 \mathrm{rad} / \mathrm{s}$ in order to obtain the RAOs. Through the harmonic analysis using FFTs for each incident wave frequency (as demonstrated above for $6.0 \mathrm{rad} / \mathrm{s}$ ), we can decompose the harmonics at each order, with first-, second- and third-order corresponding to single-, double- and triple-frequency. Fig. 36 shows the RAOs of heave force on Barge 1 at first, second and third orders, with comparisons between different cases with increased wave steepnesses. As expected, the first-order forces in Fig. 36(a) generally agree better for the three steepnesses, which should be all close to the linear prediction. Second-order force RAOs in Fig. 36(b) show a considerably large variation with rather low values for the mild wave $k A=0.005$ but significant contributions for the steep wave $k A=0.1$. 


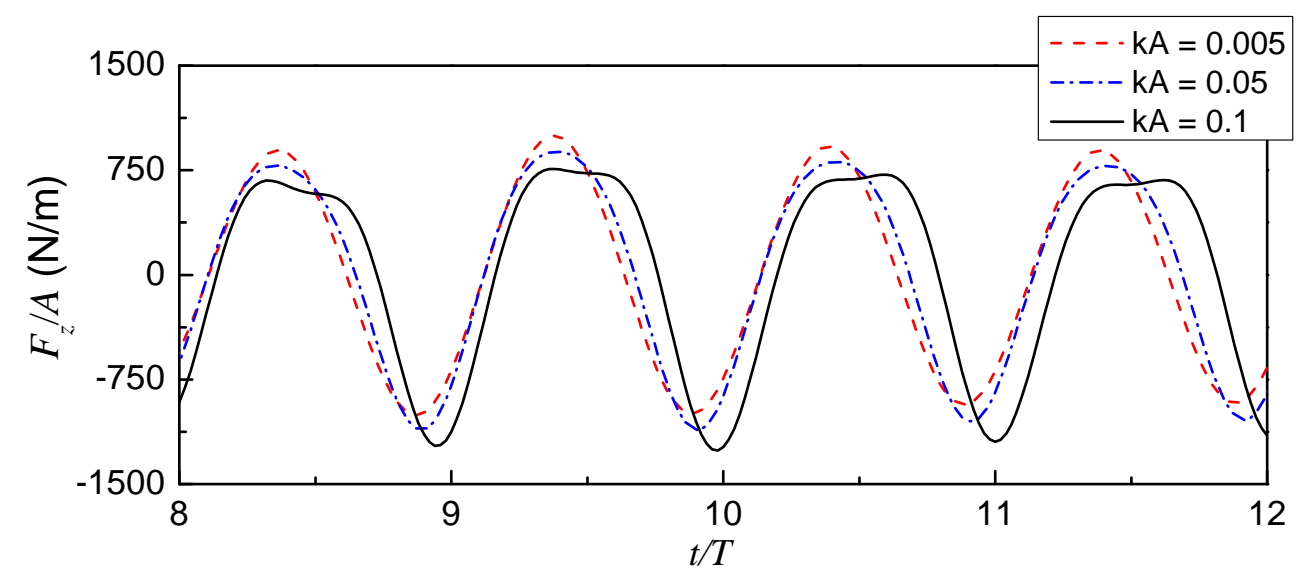

(a) Heave force

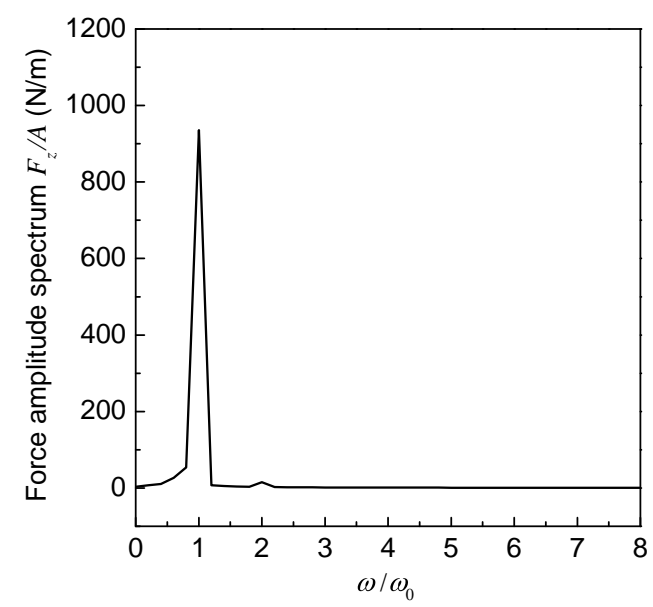

(b) $k A=0.005$

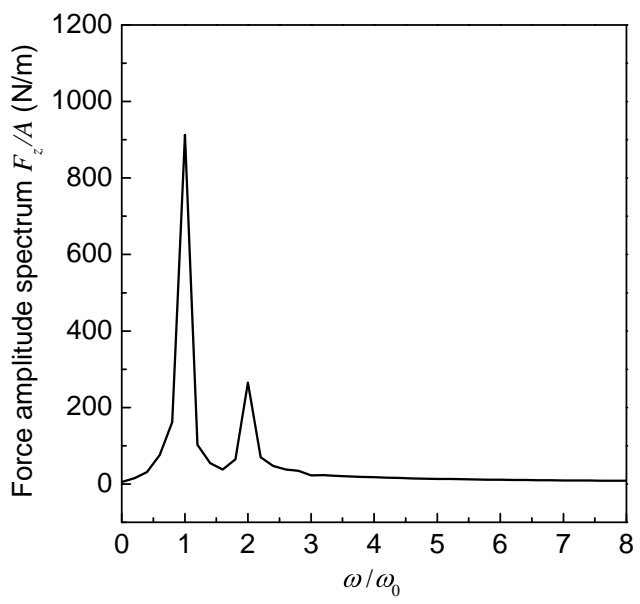

(c) $k A=0.1$

Figure 35: Heave forces on Barge 1 in the case of two middle-hinge connected barges in head sea, with incident waves of different steepnesses at frequency $\omega=6.0 \mathrm{rad} / \mathrm{s}$ : (a) time history; (b) force amplitude spectrum for $k A=0.005$ and (c) force amplitude spectrum for $k A=0.1$

The second-order forces in the case $k A=0.05$ are mostly between those in $k A=0.005$ and $k A=0.1$. The maximum second-order force in $k A=0.1$ appears near $\omega=5.8 \mathrm{rad} / \mathrm{s}$, which is about $300 \mathrm{~N} / \mathrm{m}$ and over one third its corresponding first-order force. Furthermore, the second-order force in $k A=0.1$ at $\omega=7.0 \mathrm{rad} / \mathrm{s}$ is nearly half the corresponding first-order force. These have highlighted the importance of the second harmonic component in the case where the middle-hinge connected multibody system is subject to a relatively steep wave.

While the second-order forces contribute greatly to the nonlinear responses in steep waves, the third-order components in this case play a quite minor role having a magnitude one order lower than the second-order components, as shown in Fig. 36(c) Both the waves in the cases of $k A=0.005$ and $k A=0.05$ give very low third-order heave forces. The peak third-order response for $k A=0.1$ appears at $\omega=6.0 \mathrm{rad} / \mathrm{s}$, which 
is actually negligible comparing with the first- and second-order forces. This is seen from the heave force amplitude spectrum in Fig. 35(c), Comparisons of simulation results using increased wave steepness clearly demonstrate the nonlinear effect associated with the nonlinear free surface conditions. The harmonic analysis shows that in the middle-hinge connection case the second harmonic component is significant and contributes much to the nonlinear responses, and the higher harmonics play a minor role. This further suggests that a second-order model would remarkably improve the accuracy of linear predictions, for the case here.

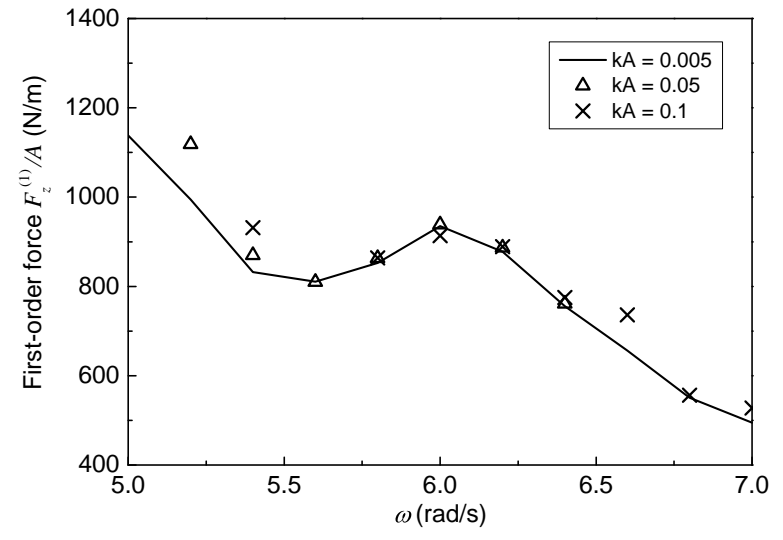

(a) First harmonic

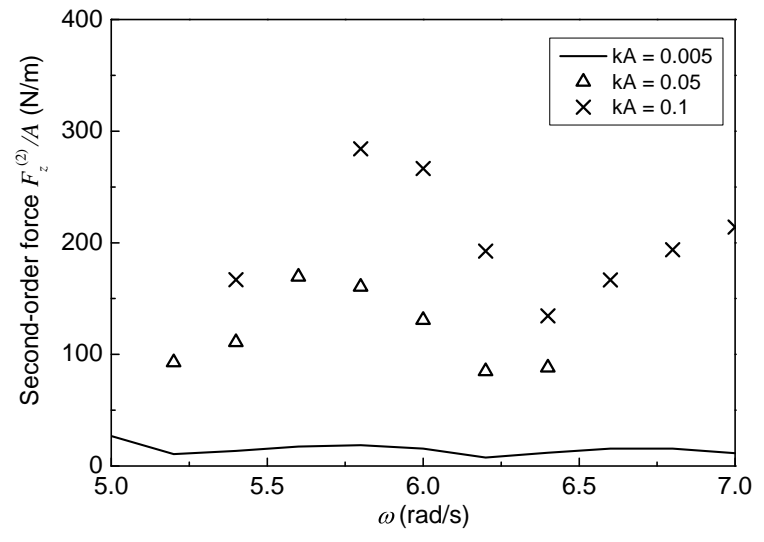

(b) Second harmonic

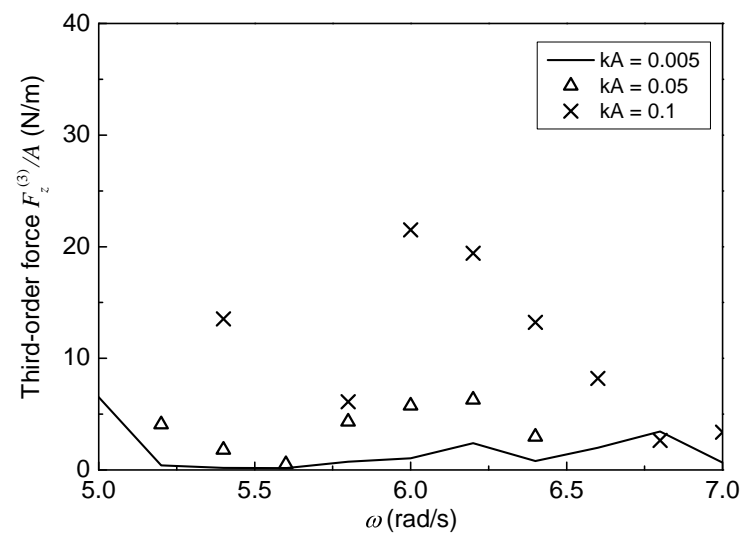

(c) Third harmonic

Figure 36: Higher-harmonic heave forces on Barge 1 in the case of two middle-hinge connected barges in head sea, with incident waves of different steepnesses: (a) First harmonic; (b) Second harmonic and (c) Third harmonic

\section{Conclusions}

This piece of work presents a fully nonlinear decomposition model which is aimed at simulating wave interaction with multiple floating bodies. We have in this study extended the application of auxiliary function approach for indirect calculation of hydrodynamic forces for multiple moving bodies. As comparison to a single body, the influence of the presence of multiple bodies can be mathematically identified from the 
resultant coupled motion equations. In the case of two bodies, the present model does not solve two separate sets of equations of 6 DOFs, but a set of 12 equations which describe the wave-multibody interactions. In the case where interconnections exist, to mathematically represent the interconnections, we impose a constraint matrix to describe different types of connections. The motion equations are then modified correspondingly to solve the constraint forces simultaneously. The proposed constraint matrix method is demonstrated to be applicable to different connection types.

Two freely moving, asymmetrically arranged barges subject to a $0^{\circ}$ heading wave have been firstly simulated to validate the proposed model. Mesh convergence is confirmed by comparing simulation results of three mesh configurations. It is as expected observed that a significant drift effect appears in the surge, sway and yaw motions of the barges, of which directions there is no restoring force/moment. In order to perform a harmonic analysis, the drift effect is eliminated by subtracting the component due to the mean acceleration which is actually a result of mean drift force. After eliminating the drift effect, the FFT can be applied to extract each harmonic component. Comparisons of first-order forces and motions are made with the linear commercial software HydroStar ${ }^{\circledR}$. A satisfactory agreement is achieved.

Further validation is performed for two tandem connected barges, as studied in Newman (1994). The comparisons between the present model and Newman's modal expansion model show very good agreements, in both rigid and hinged connections. We then investigate two side-by-side interconnected barges in both head sea and beam sea. Two types of connections are considered, which are rigid connection and middlehinge connection. The barge responses are compared with two freely floating barges to examine the influence of the connections. In the rigid connection (a horizontal bar), the two barges perform as two components of a single body. In head sea, the sway, roll and yaw motions of each barge become nearly zero because of symmetry. An in-line constraint force in the rigid bar is induced as expected. A more striking constraint force is the bending moment with respect to the $z$-axis, $M_{z}$, due to the relative yaw motions of the two barges. This significant bending moment on the connection could in practice be a critical consideration for the rigid connection. In beam sea, the roll motions of both upwave and leeside barges are effectively suppressed by the rigid connection. However, the in-line force in the rigid connection is dramatically increased since it coincides with the wave direction, and the large bending moment in the roll direction $M_{x}$ is also of great concern.

The possibly large constraint moments are relieved in the case of hinge(s) connection. In the symmetric configuration in head sea, the yaw drift is found to be reduced in the case of middle-hinge connection, comparing with two freely floating barges. In beam sea, generally the presence of the hinge connection helps to diminish the discrepancy of motions between the upwave and leeside barges, i.e. suppressing the motion of the upwave barge and enlarging that of the leeside barge.

The advanced feature of the present nonlinear modelling is demonstrated by simulations using steeper waves. Comparisons with mild wave cases show clearly the nonlinear responses of wave elevations, wave forces and body motions. Through harmonic analysis of the nonlinear responses, higher-order harmonics 
are decomposed to demonstrate the nonlinear effect. It is found that in the case of middle-hinge connection the second-order responses contribute much to the nonlinearity and third- or even higher-order components play a minor role. This might suggest that a second-order simulation would greatly improve the accuracy of linear models in this case.

Lastly, it ought to be made clear that more connection types can be modeled using the constraint matrix approach presented in this study. It definitely would be more valuable to consider some more realistic cases such as spring-moored barges. Nonetheless, the value of this work lies in the demonstration of effectiveness of the newly derived coupled auxiliary functions as well as the constraint matrix approach. The capability of nonlinear simulation is also an advanced feature of the present numerical model.

\section{References}

Bai, W., Eatock Taylor, R., 2006. Higher-order boundary element simulation of fully nonlinear wave radiation by oscillating vertical cylinders. Applied Ocean Research 28, 247 - 265. doi:http://dx.doi.org/10.1016/ j.apor.2006.12.001

Bai, W., Feng, X., Eatock Taylor, R., Ang, K., 2014. Fully nonlinear analysis of near-trapping phenomenon around an array of cylinders. Applied Ocean Research 44, 71 - 81. doi http://dx.doi.org/10.1016/j. apor.2013.11.003.

Choi, Y., Hong, S., 2002. An analysis of hydrodynamic interaction of floating multi-body using higher-orderboundary element method. Proceedings of 12th International Offshore and Polar Engineering Conference (ISOPE), Kitakyushu, Japan, 2002, $303-308$.

Cointe, R., Geyer, P., King, B., Molin, B., Tramoni, M., 1990. Nonlinear and linear motions of a rectangular barge in a perfect fluid. Proceedings of the 18th ONR Symposium on Naval Hydrodynamics, 85 - 99.

Cummins, W.E., 1962. The impulse response function and ship motions. report 1661. Department of the Navy, David Taylor Model Basin, Washington .

Fang, M.C., Kim, C.H., 1986. Hydro dynamically coupled motions of two ships advancing in oblique waves. Journal of Ship Research 30, 159 - 171.

Feng, X., Bai, W., 2015. Wave resonances in a narrow gap between two barges using fully nonlinear numerical simulation. Applied Ocean Research 50, 119 - 129. doi http://dx.doi.org/10.1016/j.apor.2015.01. 003.

Fenton, J., 1988. The numerical solution of steady water wave problems. Computers and Geosciences 14, 357 - 368. doi http://dx.doi.org/10.1016/0098-3004(88)90066-0.

Ferrant, P., 1993. Three-dimensional unsteady wave-body interactions by a rankine boundary element method. Ship Technology Research 40, 165 - 175. 
Fu, S., Moan, T., Chen, X., Cui, W., 2007. Hydroelastic analysis of flexible floating interconnected structures.

Ocean Engineering 34, 1516 - 1531. doi http://dx.doi.org/10.1016/j.oceaneng.2007.01.003.

Hong, S., Kim, J., Cho, S., Choi, Y., Kim, Y., 2005. Numerical and experimental study on hydrodynamic interaction of side-by-side moored multiple vessels. Ocean Engineering 32, 783 - 801. doi:http://dx.doi. org/10.1016/j.oceaneng.2004.10.003.

Kashiwagi, M., Endo, K., Yamaguchi, H., 2005. Wave drift forces and moments on two ships arranged side by side in waves. Ocean Engineering 32, 529 - 555. doi http://dx.doi.org/10.1016/j.oceaneng. 2004. 09.005

Kodan, N., 1984. The motion of adjacent floating structure in oblique waves. Proceeding of the 3rd Offshore Mechanics and Arctic Engineering Conference 1, 206 - 213.

Koo, B.J., Kim, M.H., 2005. Hydrodynamic interactions and relative motions of two floating platforms with mooring lines in side-by-side offloading operation. Applied Ocean Research 27, 292 - 310. doi http: //dx.doi.org/10.1016/j.apor.2006.02.001.

Koo, W., Kim, M.H., 2004. Freely floating-body simulation by a 2d fully nonlinear numerical wave tank. Ocean Engineering 31, 2011 - 2046. doi http://dx.doi.org/10.1016/j.oceaneng.2004.05.003.

Lee, C.H., Newman, J.N., 2000. An assessment of hydroelasticity for very large hinged vessels. Journal of Fluids and Structures 14, 957 - 970. doi http://dx.doi.org/10.1006/jfls.2000.0305.

Loken, A.E., 1981. Hydrodynamic interaction between several floating bodies of arbitrary form in waves. Proceeding of the International Symposium on Hydrodynamics in Ocean Engineering 2, $745-779$.

Newman, J., 1994. Wave effects on deformable bodies. Applied Ocean Research 16, 47 - 59. doi http: //dx.doi.org/10.1016/0141-1187(94)90013-2

O'Cathain, M., Leira, B.J., Ringwood, J.V., Gilloteaux, J.C., 2008. A modelling methodology for multibody systems with application to wave-energy devices. Ocean Engineering 35, 1381 - 1387. doi http: //dx.doi.org/10.1016/j.oceaneng.2008.05.005.

Ohkushu, M., 1974. Ship motions in vicinity of a structure. Proceedings of the International Conference on Behavior of Offshore Structure 1, $284-306$.

Sun, L., Eatock Taylor, R., Choo, Y.S., 2011. Responses of interconnected floating bodies. The IES Journal Part A: Civil \& Structural Engineering 4, 143 - 156. doi http://dx.doi.org/10.1080/19373260.2011. 577933.

Taghipour, R., Moan, T., 2008. Efficient frequency-domain analysis of dynamic response for the multi-body wave energy converter in multi-directional waves. Proceedings of 18th International Offshore and Polar Engineering Conference (ISOPE), Vancouver, Canada, 2008 . 
Tajali, Z., Shafieefar, M., 2011. Hydrodynamic analysis of multi-body floating piers under wave action. Ocean Engineering 38, 1925 - 1933. doi/http://dx.doi.org/10.1016/j.oceaneng.2011.09.025.

Tanizawa, K., 1995. A nonlinear simulation method of 3-d body motions in waves (1st report). Journal of the Society of Naval Architects of Japan 1995, 179-191. doi 10.2534/jjasnaoe1968.1995.178_179

Van Oortmerssen, G., 1979. Hydrodynamic interaction between two structures of floating in waves. Proceedings of BOSS'79 2nd International Conference on Behavior of Offshore Structures, 339 - 356.

Vinje, T., Brevig, P., 1981. Numerical simulation of breaking waves. Advances in Water Resources 4, 77 82. doi http://dx.doi.org/10.1016/0309-1708(81)90027-0.

Wang, C., Tay, Z., Gao, R., Koh, C., 2010. Hydroelastic response of very large floating structure with a flexible line connection. Proceedings of 29th International Conference on Ocean, Offshore and Arctic Engineering (OMAE), Shanghai, China, 2010 , $203-211$.

Wu, G.X., Eatock Taylor, R., 2003. The coupled finite element and boundary element analysis of nonlinear interactions between waves and bodies. Ocean Engineering 30, 387 - 400. doi http://dx.doi.org/10. 1016/S0029-8018(02) 00037-9. 